\title{
Structural Approaches to Control Interlayer Interactions in 2D Covalent Organic Frameworks
}

\author{
Marta Martínez-Abadía $^{[\mathrm{a}]}$ and Aurelio Mateo-Alonso ${ }^{[\mathrm{a}, \mathrm{b}] *}$ \\ ${ }^{[a]}$ POLYMAT, University of the Basque Country UPV/EHU, Avenida de Tolosa 72, E-20018 \\ Donostia-San Sebastian,Spain.Email:amateo@polymat.eu \\ ${ }^{[b]}$ Ikerbasque, Basque Foundation for Science, Bilbao, Spain.
}

\begin{abstract}
Our ability to design and synthesise monomers can affect fundamental aspects in two-dimensional covalent organic frameworks, such as dimensionality, topology and pore size. Besides this, the structure of the monomers can also affect interlayer interactions, which provide an additional means to influence crystallinity, layer arrangement, interlayer distances, and exfoliability. This Progress Report illustrates some of the effects that the structure of monomers can have on the interlayer interactions in two-dimensional covalent organic frameworks and related materials.
\end{abstract}




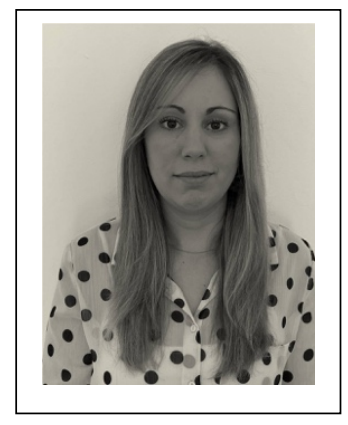

Marta Martínez-Abadía received her B.S. degree from the University of Zaragoza, Spain in 2010. She obtained her Ph.D. degree in Chemistry with Prof. M. Blanca Ros and Dr. Raquel Giménez, where she worked on the area of luminescent bent-core liquid crystals. She joined Prof. Aurelio MateoAlonso's group as a postdoctoral researcher at POLYMAT in 2016. Her current research interests are focused on the synthesis of covalent organic frameworks and supramolecular systems.

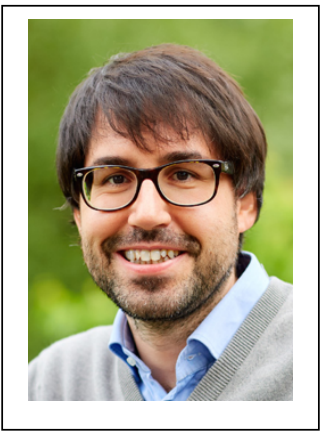

Aurelio Mateo-Alonso (Koke) obtained his BSc (1999) and MSc (2000) degrees in Organic Chemistry from Universidad Autónoma de Madrid (Spain). In 2004, he completed his PhD at Queen Mary, University of London (UK). Between 2004 and 2009, he was a Postdoctoral Researcher at Università di Trieste (Italy). In 2009, he joined the Freiburg Institute for Advanced Studies (Germany) as an independent Group Leader. Since 2012, he is an Ikerbasque Research Professor at POLYMAT and at the University of the Basque Country in San Sebastian (Spain). His research interests include organic materials synthesis, supramolecular chemistry, conjugated 2D polymers and covalent organic frameworks. 


\section{Introduction}

In 2005, the first crystalline framework constituted by covalently-bonded organic components was reported by Yaghi and co-workers. ${ }^{[1]}$ Since then, such covalent organic frameworks (COFs) have received a lot of attention from different perspectives including synthetic, structural, mechanistic, supramolecular, fundamental properties and materials development. ${ }^{[2,3,4,5,6]}$

Among these, two-dimensional COFs (2D COFs), constituted by periodically stacked 2D polymer sheets, have shown a great potential for gas separation and storage, liquid filtration and purification, heterogeneous catalysis, sensing, electronics and, energy conversion and storage applications. ${ }^{[4,7]}$ 2D COFs offers several advantages in comparison to other materials:

- 2D COFs are obtained from organic monomers that act as nodes and linkers and that can be designed at will. Therefore, by controlling the monomer structure, geometry and stoichiometry, 2D COFs with different but predictable lattices in terms of topology, dimensionality and pore size can be obtained.

- 2D COFs are crystalline as they are obtained under dynamic covalent chemistry conditions. This implies that, during COF synthesis, the covalent bonds between the monomers can be formed and broken and any defect in the framework can be repaired under thermodynamic control, providing crystalline materials.

- 2D COFs are porous. Piling-up holey 2D polymers in a periodic fashion gives rise to porous channels, whose structure will depend on both the lattice and the packing.

A high degree of crystallinity is an essential aspect in $2 \mathrm{D}$ COFs as many properties, such as porosity, homogeneity, and electronic and excitonic transport, depend strongly on long-range order. Furthermore, a high degree of crystallinity improves the ease of not only the screening and optimization of reaction conditions but also the ability to establish the structure of the resulting $2 \mathrm{D}$ 
COFs. Yet, most 2D COFs exhibit moderate crystallinity because they are produced as microcrystalline powders with small domain size and crystal defects. Different approaches have been explored to increase the crystallinity of 2D COFs. Nevertheless, it should be noted that these approaches cannot be considered as individual strategies independent from each other, but rather as factors that altogether regulate the crystallinity of 2D COFs during synthesis. These include:

- Condition-dependent approaches, which rely on controlling experimental parameters that affect the crystallinity (solvents, solvent ratio, temperature, pressure, catalysts, modulators, monomer exchange and monomer feeding rate).

- Reactivity-dependent approaches, which depend on the use of different linkages ${ }^{[3,6]}$ (boroxine, boronate ester, imine, hydrazone, azine, stilbene, etc.) that allow finding an optimal balance between crystallinity and stability. In general, more reversible reactions lead to more crystalline materials but they are often accompanied by a lower chemical stability. Other reactivity-dependent approaches involve the combination of reversible and irreversible steps ( $\beta$-ketoenamines), or the post-synthetic modification of the linkages. ${ }^{[5,6]}$ In a few cases, COFs have also been produced from irreversible reactions. ${ }^{[8]}$

- Structural-dependent approaches, which exploit structural features of the monomers that induce self-assembly. Examples of these are the introduction of molecular recognition motifs in the monomers (hydrogen bonding, donor-acceptor interactions, $\pi$ - $\pi$ stacking, etc.) or self-complementary monomers (or stackable monomers), which guide the stacking of COF layers during the polymerization and growth processes.

One of the advantages of structural-dependent approaches is our ability to design and synthesise monomers. This is because certain properties can be encoded in the monomers that afterwards are translated and even maximised in the resulting 2D COFs. Interlayer interactions, which play a key role in a broad range of properties such as crystallinity, stability, packing mode, and exfoliability of 
2D COFs, are dominated by the structure of the monomers. For instance, strongly interacting monomers generally provide 2D COFs with strongly interacting layers that favour long-range order, while weakly interacting monomers generally provide 2D COFs with weakly interacting layers that are more sensitive to solvents, vapours and mechanical friction, and also prone to exfoliation. Furthermore, by controlling the structure of the monomers it is also possible to influence the topology and to produce changes in the stacking modes of the different layers, which affect the porosity and consequently the functionality of the material.

In this Progress Report, we will cover some representative examples that illustrate how interlayer interactions in 2D COFs and related materials can be enhanced or disrupted by the structure of the monomers. For this purpose, this Report is organised in sections that encompass different structural approaches (Figure 1) that influence interlayer interactions, including monomer and layer planarization, shape-persistent monomers, and monomers encoded with attracting and disrupting interactions, illustrating how monomer design can influence the properties of 2D COFs.

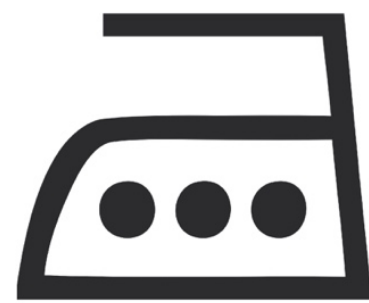

Planarization of monomers and layers

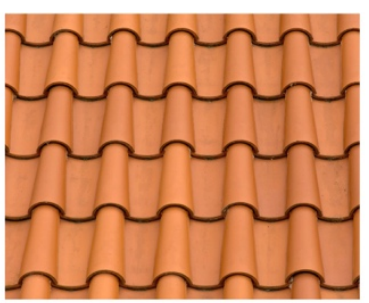

Shape-persistent monomers

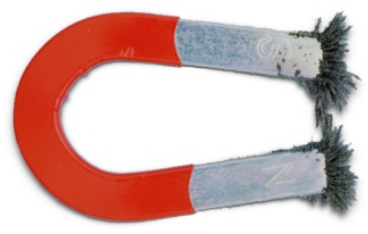

Monomers encoded with attractive interactions

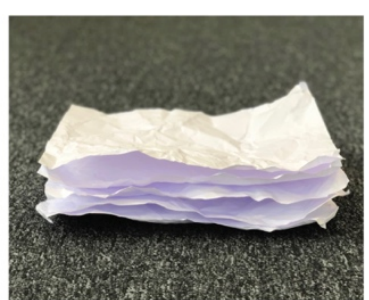

Monomers encoded with disruptive interactions

Figure 1. Representation of some of the different structural approaches that influence interlayer interactions. 


\section{Planarization of monomers and layers}

The incorporation of monomers with a large $\pi$-surface area in $2 \mathrm{D}$ COFs is known to favour interlayer $\pi-\pi$ stacking. ${ }^{[9]}$ However, the presence of substituents in such aromatic monomers can interfere with $\pi-\pi$ stacking. There are several examples that illustrate the positive relationship between planarization and 2D COF crystallinity.

A report from Zhao and co-workers ${ }^{[10]}$ have described the synthesis of three isoreticular $2 \mathrm{D}$ iminelinked COFs (N-COF, P-COF and T-COF) that were obtained by the condensation of 1,3,5benzenetricarbaldehyde with three different triamine monomers, which exhibit different planarity and flexibility (TAPA, TAPB and TAPT, Figure 2a). TAPA is non-planar because of the steric interactions between the hydrogens of the different benzene residues, while this steric interaction is reduced in TAPB leading to a slightly planarised monomer. TAPT is virtually planar because central benzene ring has been exchanged by a triazine, eliminating the steric interactions between adjacent hydrogens. As a result, T-COF with planar TAPT nodes showed sharper PXRD peaks and a closer stacking distance (3.49 $\AA$ ) than those containing non-planar building blocks (4.00 and 3.62 $\AA$ respectively for $\mathrm{N}-\mathrm{COF}$ and $\mathrm{P}-\mathrm{COF}$ ). Also, higher BET surface areas of $1149 \mathrm{~m}^{2} \mathrm{~g}^{-1}$ were observed for T-COF (692 and $435 \mathrm{~m}^{2} \mathrm{~g}^{-1}$ respectively for $\mathrm{N}-\mathrm{COF}$ and P-COF). Furthermore, the role of the monomer planarity affected also the gas uptake and separation performance of the resulting COFs. Specifically, T-COF showed the highest hydrocarbon uptake $\left(\mathrm{CH}_{4}\right.$ and $\left.\mathrm{C}_{2} \mathrm{H}_{6}\right)$ while $\mathrm{N}-\mathrm{COF}$, with the smallest pore size, showed the highest $\mathrm{C}_{2} \mathrm{H}_{6} / \mathrm{CH}_{4}$ separation. 
a)

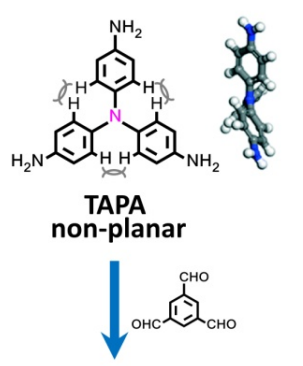

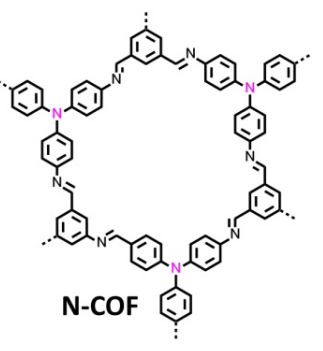

b)

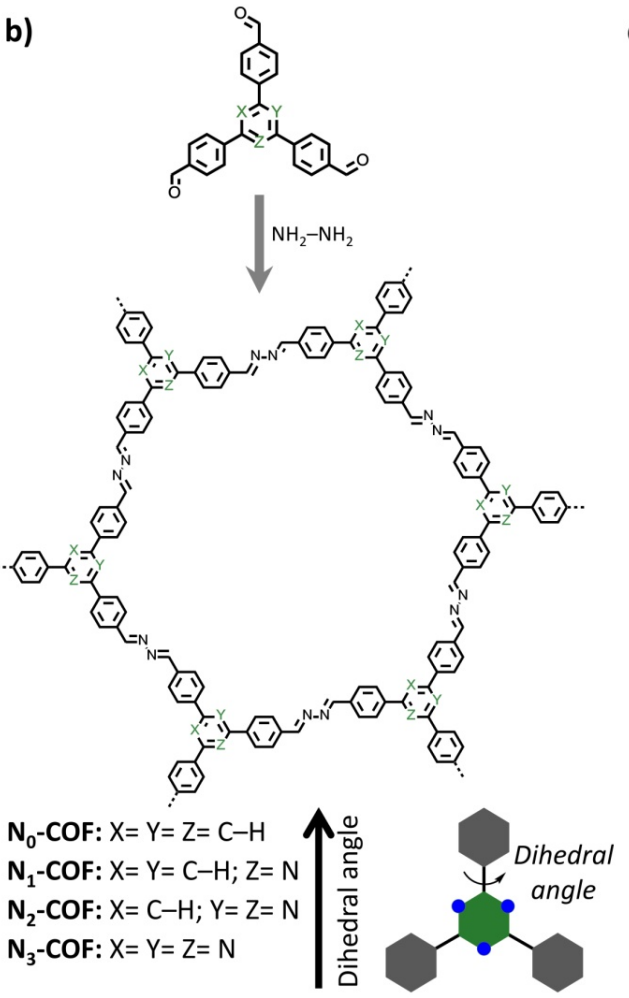

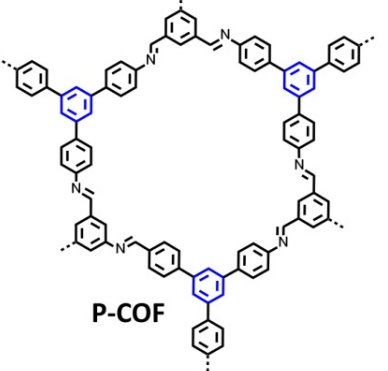

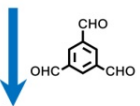<smiles>CCC(C)C(C)C</smiles>

c)

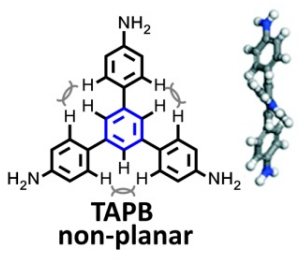

会

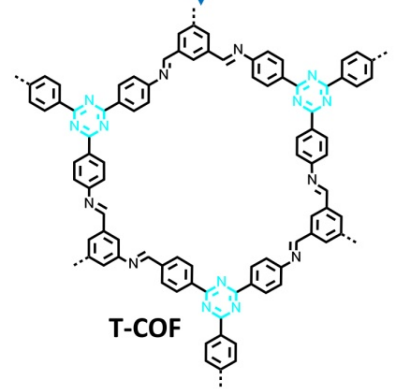

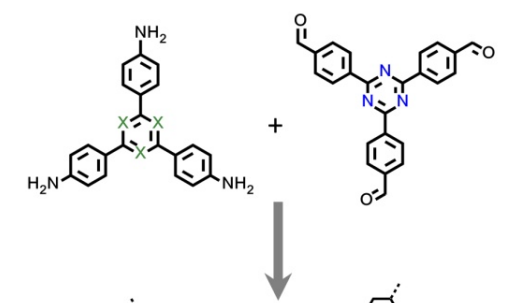

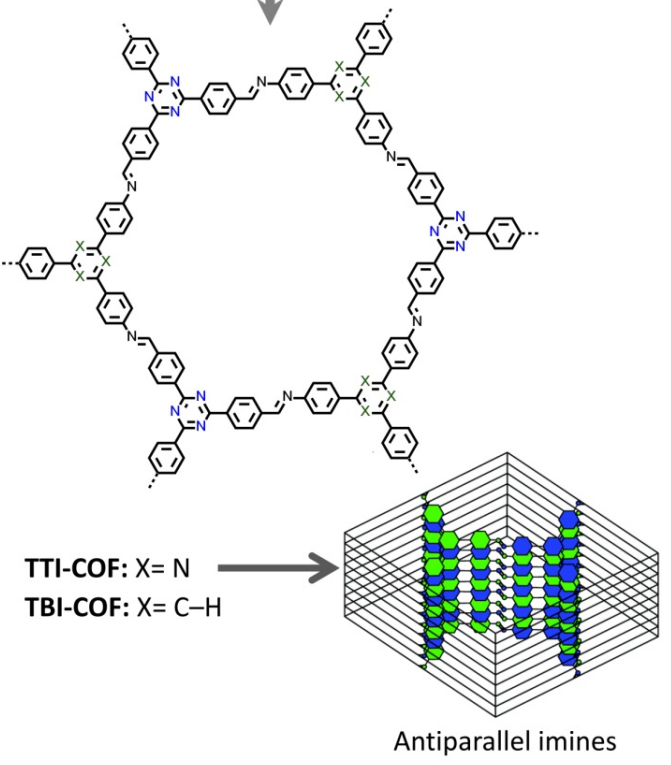

Figure 2. a) Synthesis of three isoreticular 2D COFs from 1,3,5-benzenetricarbaldehyde with various amine monomers. Adapted with permission. ${ }^{[10]}$ Copyright 2017, The Royal Society of Chemistry. b) Synthesis of $\mathrm{N}_{\mathrm{x}^{-}}$ COFs from $\mathrm{N}_{\mathrm{x}}$-aldehydes and hydrazine and representation of a triphenylarene platform. Adapted with permission. ${ }^{[11]}$ Copyright 2015, Nature Publishing Group. c) Synthesis of TTI-COF and TBI-COF and the antiparallel stacking of TTI-COF. Adapted with permission. ${ }^{[12]}$ Copyright 2017, The Royal Society of Chemistry.

Lotsch and co-workers have reported a series of azine-linked COFs $\left(\mathrm{N}_{\mathrm{x}}-\mathrm{COF}, \mathrm{x}=0,1,2\right.$ and 3) from hydrazine and triphenylarene aldehydes with the central aryl ring containing different number 
of nitrogen atoms (Figure 2b). ${ }^{[11]}$ The introduction of nitrogen atoms decreased the dihedral angle along the series, ranging from non-planar to planar geometries $\left(\mathrm{N}_{3}-\mathrm{COF}\right)$. The results demonstrated that both monomer planarity and symmetry (related with the number of nitrogen atoms) affected the crystallinity and porosity of the final $2 \mathrm{D}$ COFs. From $\mathrm{N}_{0}-\mathrm{COF}$ to $\mathrm{N}_{3}-\mathrm{COF}$ the PXRD peaks became sharper and BET surface areas of 702, 326, 1046 and $1537 \mathrm{~m}^{2} \mathrm{~g}^{-1}$ were observed for $\mathrm{N}_{0^{-}}, \mathrm{N}_{1^{-}}, \mathrm{N}_{2^{-}}$ and $\mathrm{N}_{3}-\mathrm{COF}$, respectively. When one nitrogen atom was incorporated in each peripheral aryl rings of the monomer (phenyl tripyridine core), a poorly crystalline COF was observed (PTP-COF) with a low number of reflections on the PXRD and a BET surface area of $84.21 \mathrm{~m}^{2} \mathrm{~g}^{-1},{ }^{[13]}$ which was attributed to the lower symmetry and planarity of this system compared to the isoelectronic $\mathrm{N}_{3}-\mathrm{COF}$ with three nitrogen atoms in the central phenyl ring. This trend has also been observed in other triphenylamine 2D COFs ${ }^{[14]}$ (TPA-COF-1, TPA-COF-2 and TPA-COF-3) and carbazole-based $\mathrm{COFs}^{[15]}$ (Car-TPA, Car-TPP, and Car-TPT), where the increasing planarity and symmetry of the monomers increased the crystallinity and decreased the interlayer distance.

The combination of planarity and electronic complementarity can also influence the stacking of 2D COFs. Two imine-linked COFs, TBI-COF and TTI-COF (Figure 2c), ${ }^{[12]}$ were obtained from the planar 2,4,6-tris(4-phormylphenyl)triazines with two amine monomers of different planarity: the non-planar 1,3,5-tris(4-aminophenyl)benzene and the planar 2,4,6-tris(4-aminophenyl)triazine, respectively. The flat monomers in TTI-COF allowed an antiparallel arrangement that enabled a donor-acceptor self-complementary stacking with a uniform direction of the layer offset in each subsequent layer by alternation of the different monomers, which was not observed in TBI-COF. This resulted in a higher crystallinity with narrower PXRD peaks when compared with TBI-COF and in significantly larger domain sizes (50-200 nm for TTI-COF $v s$ 30-80 nm for TBI-COF) as observed by TEM. 
The intralayer hydrogen bond interactions between imines and adjacent - $\mathrm{OH}$ functionalities in imine-based 2D-COFs suppress the imine's torsion angle, giving rise to planarised segments and in turn to more crystalline systems. Furthermore, these hydrogen bond interactions can also protect the imine from hydrolysis, increasing the chemical stability of 2D COFs. Banerjee and co-workers have shown how intralayer hydrogen bonding in a porphyrin containing imine-linked 2D COF (DhaTph) increased the crystallinity and porosity compared to the analogue COF with methoxy groups (DmaTph) (Figure 3a). ${ }^{[16]}$ DhaTph exhibited a sharper PXRD pattern and higher BET surface area $\left(1305 \mathrm{~m}^{2} \mathrm{~g}^{-1}\right)$ when compared to DmaTph $\left(431 \mathrm{~m}^{2} \mathrm{~g}^{-1}\right)$. When hydroxyl groups were incorporated in the 2,3-positions of terephthalaldehyde (2,3-DhaTph) again an increase in the crystallinity and porosity was observed compared to the linker with methoxy groups in the same positions (2,3DmaTph). ${ }^{[17]}$ The same effect was found by Jiang and co-workers in a series of tetragonal 2D porphyrin COFs from 5,10,15,20-tetrakis(4'-tetraphenylamino)porphyrin derivatives $\left(\mathrm{M}=\mathrm{H}_{2}, \mathrm{Cu}\right.$ and $\mathrm{Ni}$ ) and a mixture of 2,5-dihydroxyterephthalaldehyde and terephthalaldehyde at different molar ratios $(100 / 0,75 / 25,50 / 50$ and $25 / 75) .{ }^{[18]}$ The results suggested that increasing the number of hydrogen bonds (molar ratio 100/0) was beneficial for the COF crystallinity and this implied better properties of the material such as light-harvesting capability, reducing the band gap, and enhancing their photocatalytic activity toward the generation of singlet oxygen. 
a)
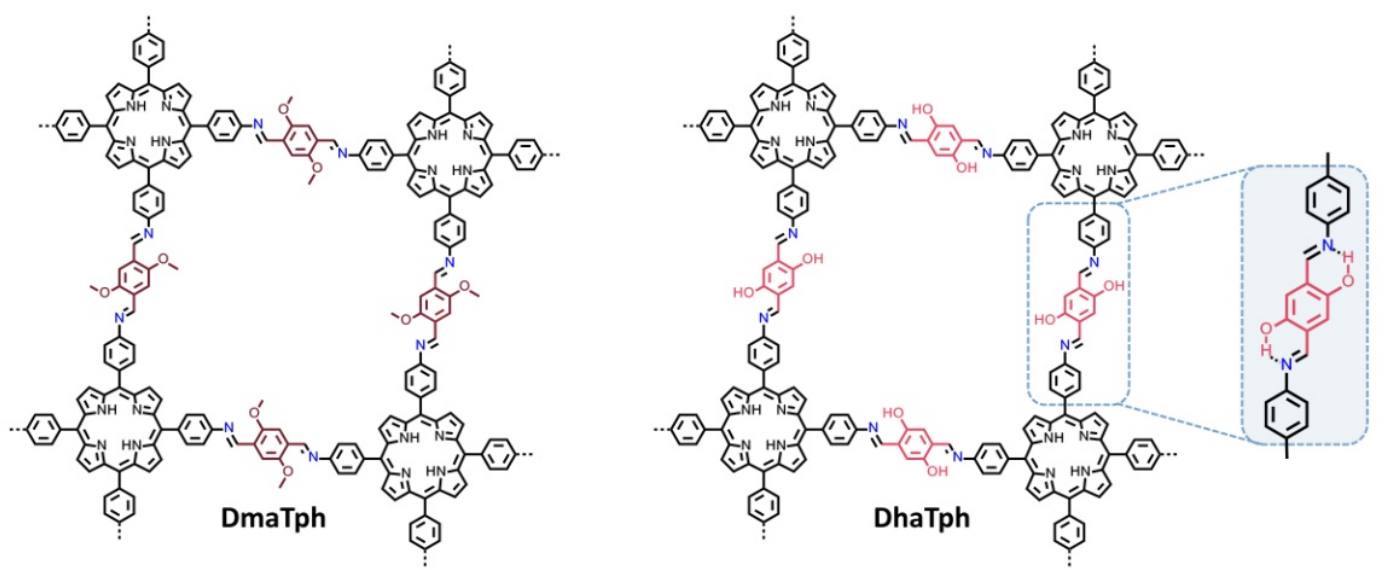

b)
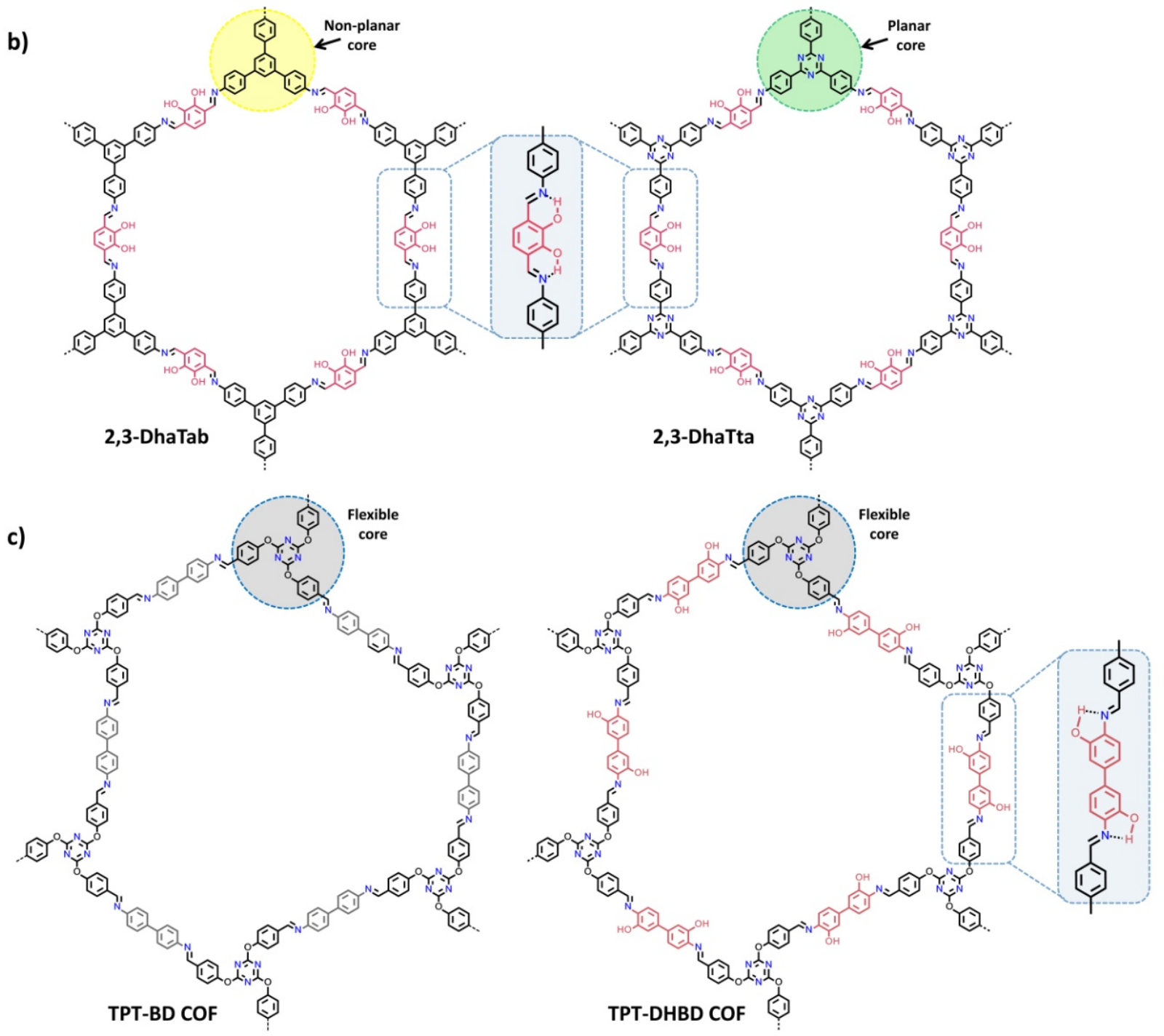

Figure 3. Chemical structure of: a) DmaTph and DhaTph; b) 2,3-DhaTab and 2,3-DhaTta; and c) TPT-BD COF and TPT-DHBD COF. 
The combination of planar triazine nodes with intralayer hydrogen bonding has also proved to be an excellent strategy for producing highly crystalline 2D COFs. For instance, the condensation of 2,5dihydroxyterephthalaldehyde gave rise to highly crystalline imine-linked COFs (2,3-DhaTta and 2,3-DhaTab), ${ }^{[19]}$ in which the hydrogen bonding locked the phenyl rings in one plane (Figure $3 b$ ). Again, the high crystallinity was attributed to the presence of strong intramolecular hydrogen bonds that increased the interlayer stacking. 2,3-DhaTta with a planar triazine node showed a higher crystallinity and BET surface area than 2,3-Dha-Tab with a non-planar node $\left(S_{\mathrm{BET}}=1700\right.$ and 413 $\mathrm{m}^{2} \mathrm{~g}^{-1}$, respectively for 2,3-DhaTta and 2,3-DhaTab). Yan and co-workers also synthesized a highly crystalline COF (TzDa) by combining a planar triazine node and intralayer hydrogen bonds. ${ }^{[20]}$

Planarization by intralayer H-bonding has also shown to be a powerful approach for preparing crystalline imine-linked COFs with flexible monomers, which is quite challenging. Crystalline 2D COFs containing flexible 2,4,6-tris(4-formylphenoxy)-1,3,5-triazine monomers (TP-DHBD-COF, Figure 3c) have been obtained by introducing 3,3'-dihydroxybenzidine residues that promote the planarization by intralayer hydrogen bonding. ${ }^{[21]}$ In fact, higher crystallinity and BET surface areas were observed in TP-DHBD-COF when compared with TP-BD-COF (Figure 3c), namely the parent COF with benzidine residues lacking intralayer hydrogen bonds. Even the combinatorial copolymerization with a mixture of benzidine and 3,3'-dihydroxybenzidine at different molar ratios $\left(\mathrm{TP}-\mathrm{DHBD}_{\mathrm{x}} \mathrm{COFs} \mathrm{x}=25,50,75\right)$ resulted in an improvement of crystallinity by increasing the proportion of dihydroxybenzidine.

The planarization of azine-linked COFs has also been achieved by intralayer hydrogen bonding. Esteves and co-workers reported the effect of a systematic increase of hydroxyl groups (from one to three) in three azine-linked COFs (RIO-11, RIO-12 and RIO-13). ${ }^{[22]}$ The study revealed that the increase of the number of hydrogen bonds enhanced crystallinity with an increasing number of hydroxyl groups, achieving sharper PXRD peaks along the series and BET surface areas of 242, 
1008 and $1205 \mathrm{~m}^{2} \mathrm{~g}^{-1}$ for RIO-11, RIO-12 and RIO-13, respectively. Also Liu and co-workers have reported COF-JLU3 ${ }^{[23]}$ obtained from hydrazine and 1,3,5-tris(3'-tert-butyl-4'-hydroxy5'formylphenyl)benzene, where the presence hydroxyl groups not only improved the crystallinity but also were able to act as cation receptors with a high sensitivity and selectivity to $\mathrm{Cu}^{2+}$.

The planarization by intralayer hydrogen bonds has also been observed in benzobisthiazole-linked COFs. ${ }^{[24]}$ The presence of hydroxyl groups in PG-BBT planarised the structure by intralayer hydrogen bonds with the nitrogens of the benzobisthiazole units, increasing its crystallinity and stability in comparison to BZ-BBT, which show the equivalent structure but without hydrogen bond donating hydroxyl groups. 


\section{Shape-Persistent Monomers}

Shape-persistent monomers can also be used to increase 2D COF crystallinity. In this context, the structural self-complementarity of monomers with different shapes, such as bowl, propeller and arm-chair, guides the stacking of COF layers during the nucleation and growth processes.

An early example by Zheng and colleagues has demonstrated how the introduction of cyclotriveratrylene (CTV), a bowl-shaped macrocycle, provides crystalline 2D COFs. CTV nodes were incorporated in a boronate ester-linked COF using 1,4-phenylenediboronic acid as linker, leading to an undulated 2D lattice (CTC-COF, Figure 4) ${ }^{[25]}$ with sharp PXRDs and high surface areas $\left(\mathrm{S}_{\mathrm{BET}}=1710 \mathrm{~m}^{2} \mathrm{~g}^{-1}\right)$. The high crystallinity was attributed to the ability of the bowls to stack in the 2D COF. CTVs have also been introduced in imine-linked COFs from the triformyl-CTV ${ }^{[26]}$ and triamine-CTV ${ }^{[27]}$ derivatives, leading to COFs with moderate crystallinity.

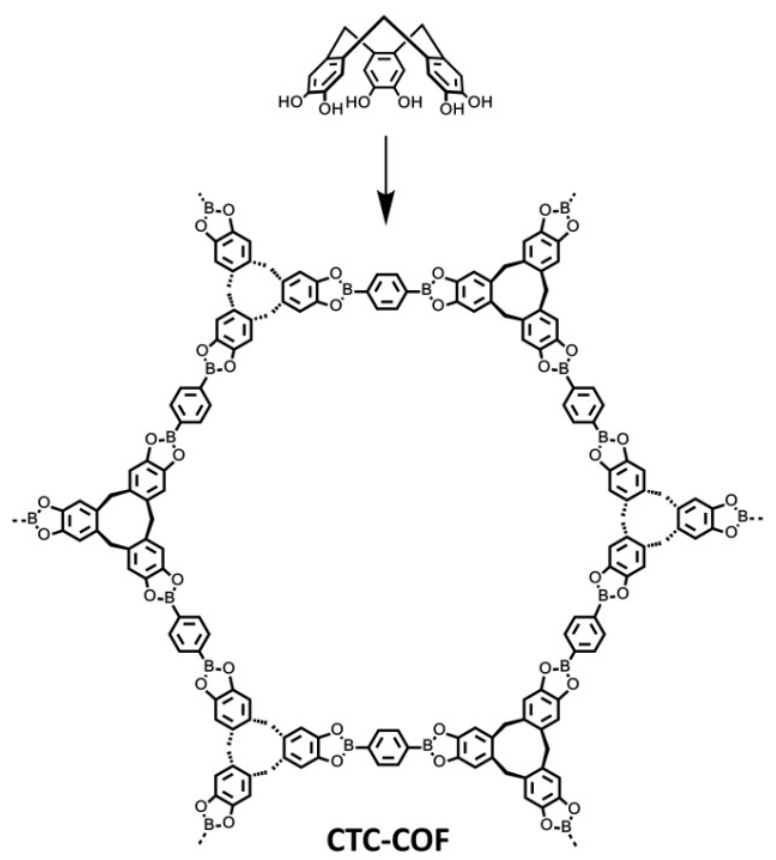

Figure 4. Synthesis of a CTV based boronate ester-linked COF (CTC-COF). 
Bein, Auras and co-workers have shown that the propeller structure of tetraphenylethylene derivatives can be beneficial to increase crystallinity in 2D COFs (Figure 5a). ${ }^{[28]}$ The phenyl groups of tetraphenylethylene can adopt several conformations but once in the $2 \mathrm{D}$ lattice it adopts a propeller conformation, defining a fixed docking site for the attachment of adjacent layers, where only propellers with the same conformation can stack on top of each other. Therefore, propellers must synchronise conformationally during 2D COF synthesis. Through this approach, highly crystalline imine-linked COFs were obtained from the condensation of 4,4',4", $4^{\prime \prime \prime}$-(ethylene-1,1,2,2tetrayl)-tetraaniline (ETTA) with four aromatic dialdehyde monomers with different symmetry and flexibility (terephthalaldehyde, biphenyl-4,4'-dicarboxaldehyde, $\quad p$-tertphenyl-4,4"'dicarboxaldehyde and thieno[3,2-b]thiophene-2,5-dicarboxaldehyde). The materials showed intense and sharp PXRD patterns, BET surface areas ranging from 1000 to $2140 \mathrm{~m}^{2} \mathrm{~g}^{-1}$ and crystalline domains sizes from 50 to $100 \mathrm{~nm}$. Besides the positive effect in crystallinity, the advantage of ETTA monomers is that they can be used to prepare 2D COFs for several applications, such as light-driven water reduction, ${ }^{[29]}$ catalysis, ${ }^{[30]}$ adsorption of heavy metals, ${ }^{[31]}$ optoelectronic applications, ${ }^{[32]}$ lithium-sulfur batteries, ${ }^{[33]}$ iodine capture, ${ }^{[34]}$ gas sorption and storage, ${ }^{[35]}$ and fluorescent probes. ${ }^{[36]}$ 

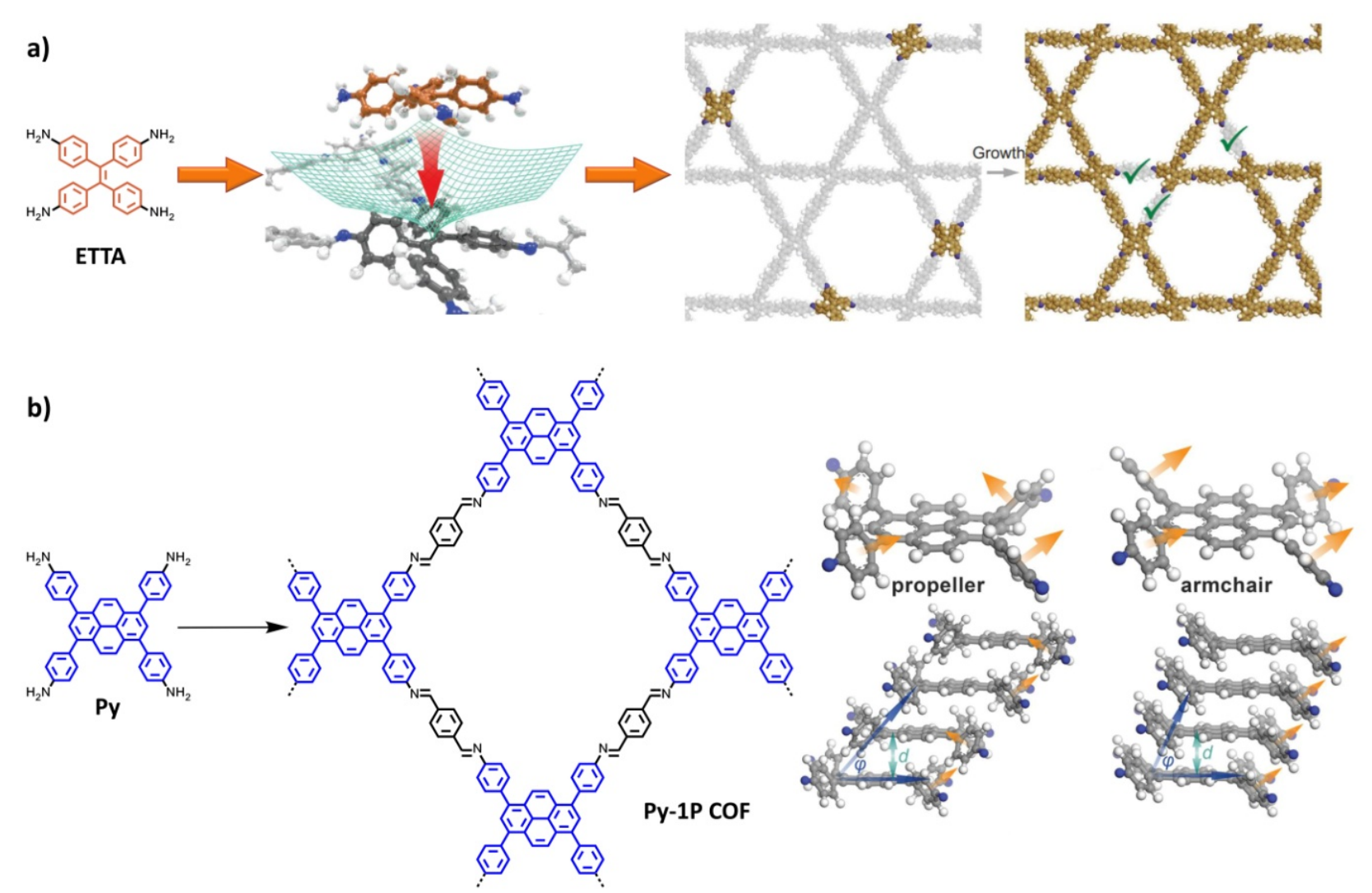

Figure 5. a) Structure of ETTA and illustration of the guided attachment of the ETTA-based building blocks. Adapted with permission. ${ }^{[28]}$ Copyright 2016, Nature Publishing Group. b) Synthetic scheme for the construction of Py-1P COF and possible molecular conformations and corresponding stacking arrangements of 1,3,6,8-tetrakis(4-aminophenyl)pyrene when incorporated into a COF. In the "propeller" conformation the normal vectors of the phenylenes describe a circle. These molecules form offset stacks of alternating leftand right-handed propellers, whereby the phenylenes arrange in an edge-on-face sequence. In the "armchair" configuration the normal vectors of the phenylenes point into the same direction. Adapted with permission. ${ }^{[37]}$ Copyright 2016, American Chemical society. 
This approach can be generalised to other monomers with several well-defined conformations, including tris(4-aminophenyl)amine and 1,3,5-tris(4-aminophenyl)benzene that show a trigonal propeller-shaped structure, ${ }^{[28]}$ and 1,3,6,8-tetrakis(4-aminophenyl)pyrene ${ }^{[37]}$ that show a rectangular geometry. In particular, 1,3,6,8-tetrakis(4-aminophenyl)pyrene can adopt both a propeller and an armchair conformation (Figure 5b). The armchair configuration leads to a smaller offset stacking compared to the propeller configuration, producing a closer interlayer stacking distance that defines the direction of the offset between two 2D layers. Thus, Py-1P COF crystallised in an armchair configuration, showing sharp PXRD reflections and very large domains up to $500 \mathrm{~nm}$. The high degree of crystallinity derived from this geometric interlocking has also been used for obtaining oriented thin films with solvatochromic response upon exposure to polar and non-polar vapours. ${ }^{[38]}$ The best response was observed in Py-TT COF, which was obtained by condensation of 1,3,6,8tetrakis(4-aminophenyl)pyrene (Py) with thieno-[3,2-b]tiophene-2,5-dicarboxaldehyde. In addition, the close and good packing of 1,3,6,8-tetrakis(4-aminophenyl)pyrene (Py) has also enabled the introduction of other functional linkers to produce highly crystalline COFs with aligned open docking sites, ${ }^{[39]}$ catalytic activity, ${ }^{[40]}$ near-infrared-absorbing properties, ${ }^{[41]} \mathrm{CO}_{2}$ sorption properties, ${ }^{[42]}$ electronic properties, ${ }^{[43-45]}$ and with high performance in Li-S batteries, ${ }^{[46]}$ among others.

The above-mentioned monomers showed a high degree of conformational freedom that required conformational synchronization during COF formation. To avoid this, Mateo-Alonso and colleagues have introduced core-twisted polycyclic aromatic hydrocarbons as rigid non-planar nodes, ${ }^{[47]}$ namely $2,3,10,11,18,19$-hexahydroxy-cata-hexabenzocoronene (HBC), in which there is no need for synchronization (Figure 6). In fact, HBC adopts a contorted and rigid structure that is conformationally locked as the result of steric congestion between the hydrogens in the peripheral benzene rings and thus, the hexabenzocoronene core does not undergo any conformational change at temperatures necessary for COF synthesis. The introduction of three cathecol groups in alternated 
positions of the contorted-hexabenzocoronene (HBC) produced a rigid and stable double-bowl structure with the cathecol groups locked above the coronene plane (Figure 6). The condensation of HBC with pyrene-2,7-diboronic acid generated a two-dimensional covalent organic framework with a wavy honeycomb (chairlike) lattice (Marta-COF-1), with sharp PXRD reflections and BET surface area $\left(1300 \mathrm{~m}^{2} \mathrm{~g}^{-1}\right)$ close to its theoretical value $\left(1570 \mathrm{~m}^{2} \mathrm{~g}^{-1}\right)$. The concave-convex selfcomplementarity of the wavy lattice of Marta-COF-1 lined up the chairlike mesopores of the individual layers into open channels, which could be imaged by high-resolution transmission electron microscopy (HR-TEM) and transmission electron tomography. Furthermore, the waviness of Marta-COF-1 did not interfere with interlayer $\pi-\pi$ stacking $(3.4 \AA$ at the pyrene linkers and $3.7 \AA$ at the HBC nodes), exhibiting charge transport properties similar to the most efficient $\pi$-stacked 2D COFs. 


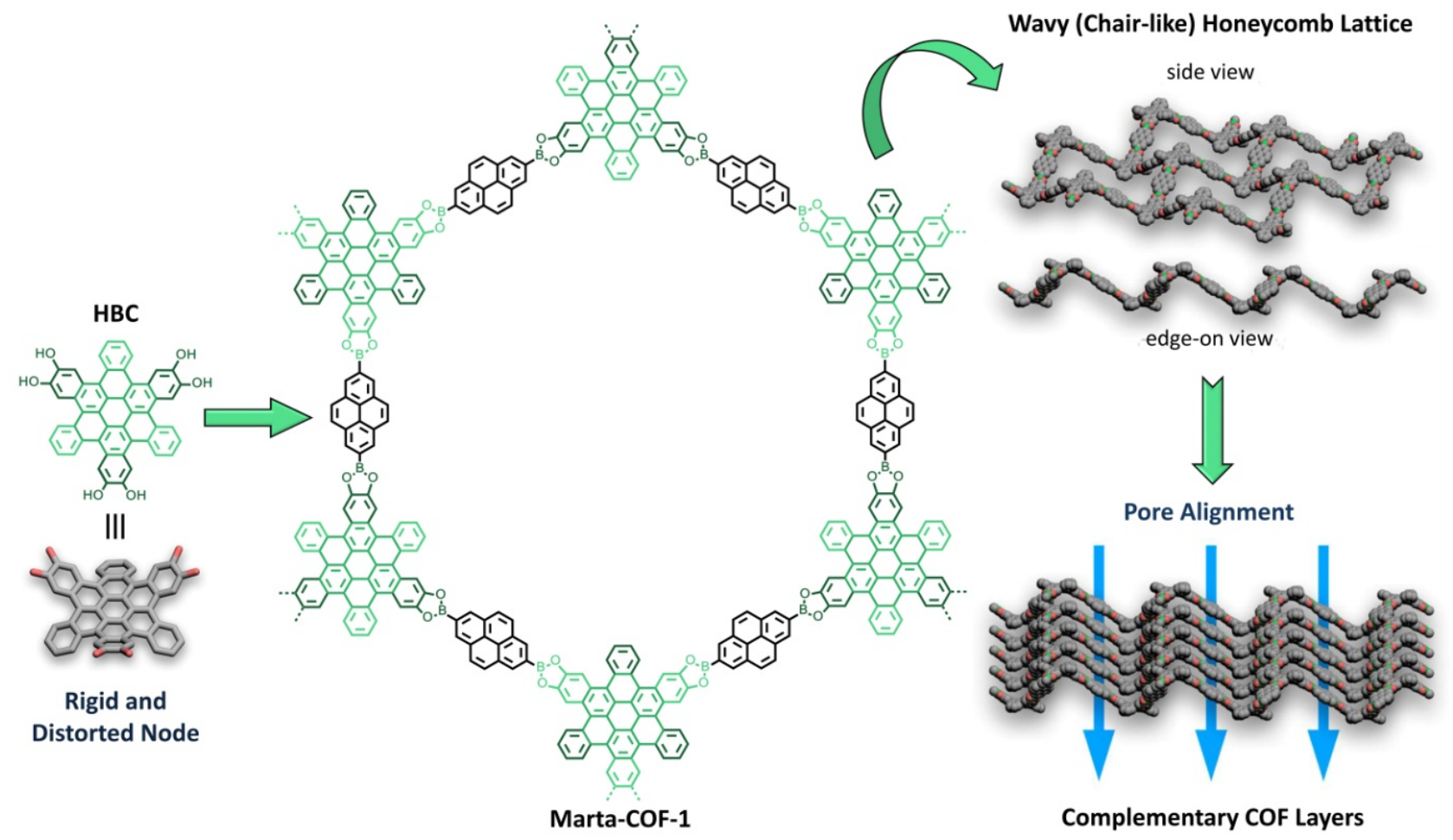

Figure 6. Schematic representation of the rigid and distorted HBC nodes and their incorporation in boronate ester COFs to obtain Marta-COF-1 with a wavy and highly complementary structure that guides the stacking of COF layers. Adapted with permission. ${ }^{[4]}$ Copyright 2019, American Chemical Society.

Chen and co-workers have explored $D_{2 \mathrm{~h}}$-symmetric dibenzo[g,p]chrysene (DBC) monomers in imine-linked COFs (DBC-TIM, Figure 7). ${ }^{[48]}$ DBC is a fused analogue of the propeller-shaped ETTA monomer, ${ }^{[28]}$ which is slightly twisted as the result of steric congestion of its cove edges. The transamination reaction of DBC-TIM and 4,4'-biphenyldicarbaldehyde produced a highly crystalline imine-linked COF (DBC-2P) with excellent stability in strong base and acid, which was attributed to the tight packing (3.6 $\AA$ ). The introduction of polyethylene glycol (PEG) and PEG$\mathrm{LiBF}_{4}$ salt into the COF channels of $\mathrm{DBC}-2 \mathrm{P}$ produced a hybrid material with high ionic conductivity. Bein, Medina and co-workers have also reported the synthesis of DBC-based 2D COFs through the direct condensation of dibenzo[g,p]chrysene-2,7,10,15-tetraamine (DBCTA, Figure 7). ${ }^{[49]}$ Its condensation with linear dialdehydes, such as terephthalaldehyde, 4,4'biphenyldicarbaldehyde and thieno[3,2-b]thiophene-2,5-dicarboxaldehyde, led to highly crystalline imine-linked COFs (DBC-COFs) with hexagonally faceted single crystals ranging from 50 to 150 
$\mathrm{nm}$. The reduced torsion of the dibenzochrysene monomer produced an interlayer stacking distance of $\sim 3.6 \AA$, which was $\sim 1 \AA$ shorter than in 2D COFs derived from ETTA. The shorter interlayer distance translated into longer photoluminescence lifetimes in DBC-COFs when compared to the ETTA-based COF analogues.

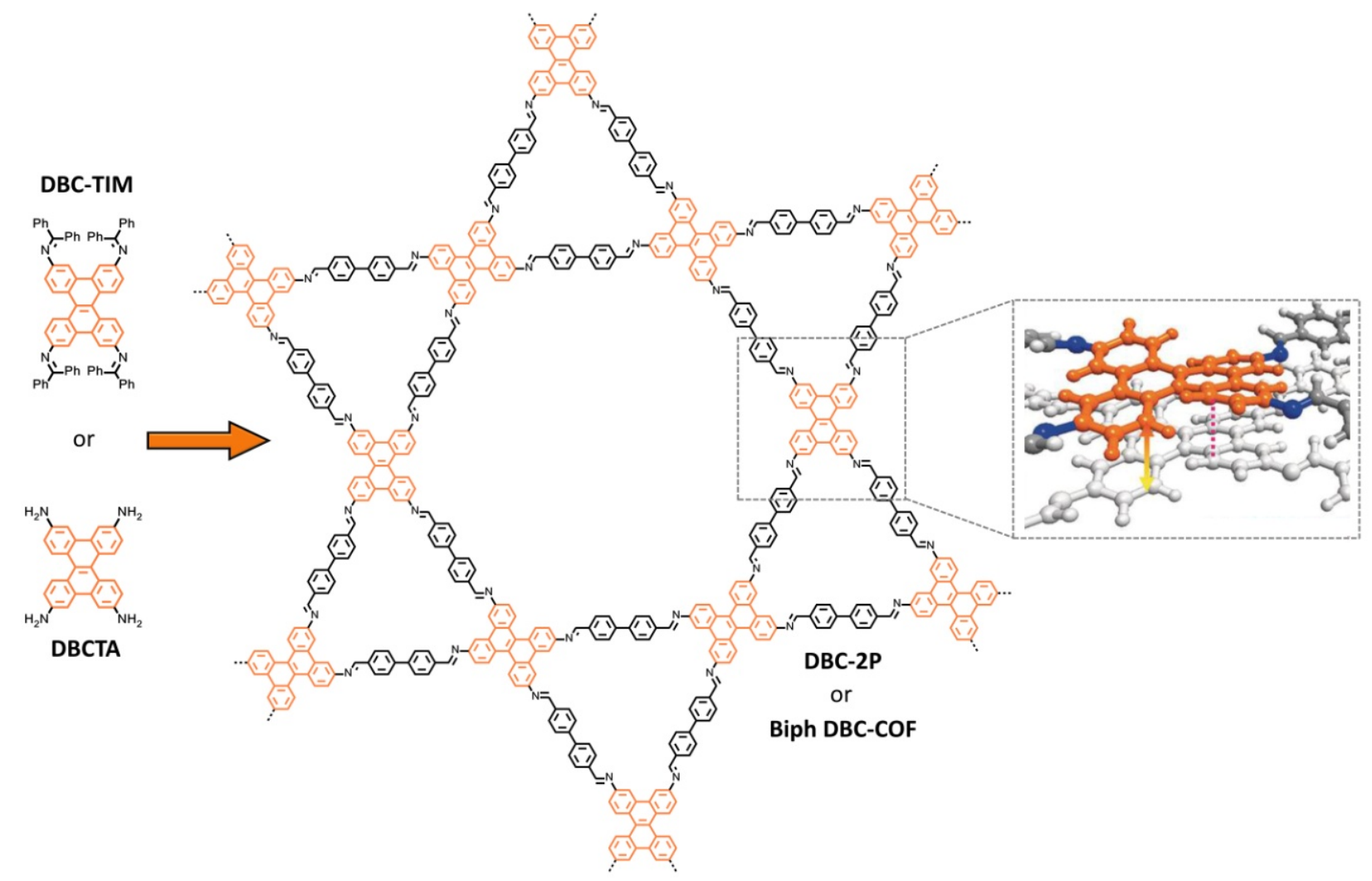

Figure 7. Synthesis of a DBC-based imine-linked COF from two different precursors (DBC-TIM and DBCTA) and the model for its stackability. Adapted with permission. ${ }^{[48]}$ Copyright 2019, John Wiley and Sons. 


\section{Monomers Encoded with Attractive Interactions}

Our ability to design monomers also allows the introduction of self-recognition patterns based on directing non-covalent interactions. Upon 2D COF formation such interactions can enhance interlayer interactions and lock the conformation between adjacent layers, increasing long range order and improving the stability.

\subsection{Dipole interactions}

Jiang and co-workers have shown how the introduction of fluorinated and unsubstituted phenylene linkers in porphyrin containing imine-linked COFs improved the crystallinity and porosity ${ }^{[50]}$ as the result of the aromatic electrostatic interaction between the fluorinated and non-fluorinated aromatic residues, which led to alternating face-to-face stacks. Five newly COFs synthesized from mixtures of 2,3,5,6-tetrafluoroterephthalaldehyde and terephthalaldehyde as linkers, at different molar ratios $(100 / 0, \quad 75 / 25, \quad 50 / 50, \quad 25 / 75$, and 0/100), with copper 5,10,15,20-tetrakis(ptetraphenylamino)porphyrin as nodes were investigated (Figure $8 \mathrm{a}$ ). The mixture 50/50 showed the most intense PXRD pattern and the highest BET surface area $\left(1389 \mathrm{~m}^{2} \mathrm{~g}^{-1}\right)$, which was consistent with the fact the electrostatic interactions between aromatics residues were maximised in a 50/50 molar ratio. Johnson and co-workers have reached similar conclusions when comparing two fluorinated imine-linked COFs, one of them partially fluorinated (FASt-COF) and the other fully fluorinated (F-COF). ${ }^{[51]}$ The results showed narrower PXRD peaks and a higher BET surface area for the partially fluorinated FASt-COF $\left(1389 \mathrm{~m}^{2} \mathrm{~g}^{-1}\right)$ in comparison to the fully fluorinated F-COF $\left(1275 \mathrm{~m}^{2} \mathrm{~g}^{-1}\right)$, which was also ascribed to the maximised stacking interactions in the presence of equal ratios of fluorinated and non-fluorinated aromatic residues. 
a)
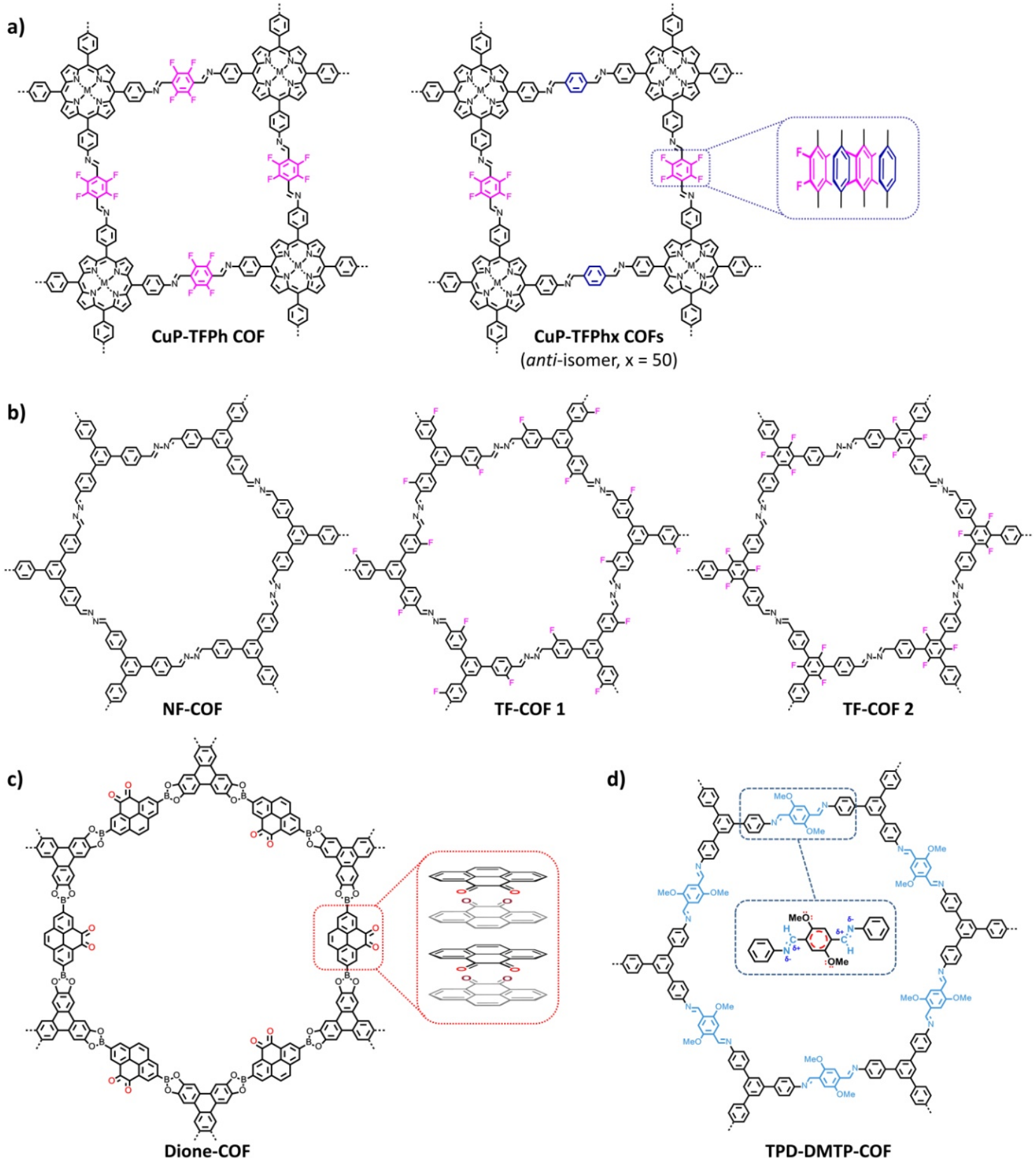

Figure 8. a) Structure of CuP-TFPh COF and CuP-TFPhx COFs; b) Schematic representation of the azine-linked COFs NF-COF, TF-COF 1 and TF-COF 2; c) Structure of Dione-COF with the columnar stacking of pyrene4,5-dione favoured by antiparallel alignment of dipole moments; d) Structure of TPD-DMTP-COF with the resonance effect of the oxygen lone pairs that weaken the polarization of the $\mathrm{C}=\mathrm{N}$ bonds and soften the interlayer repulsion in the COF. 
Smaldone and co-workers investigated the effect of electron-withdrawing substituents on the properties of azine-linked COFs (Figure 8b). ${ }^{[52]}$ The non-planar building block 1,3,5-tris(4phormylphenyl)benzene was partially substituted with fluorine atoms at the outer phenyl rings or at the core, producing 2D COFs (TF-COF 1 and TF-COF 2, respectively) with dramatically improved crystallinity, pore size and BET surface areas (1820 and $2044 \mathrm{~m}^{2} \mathrm{~g}^{-1}$, respectively) compared to the COF without fluorine substitution $\left(760 \mathrm{~m}^{2} \mathrm{~g}^{-1}\right.$ for NF-COF). The result was attributed to stronger cofacial interactions caused by the electron-withdrawing fluorine atoms. This approach was further studied by exploring azine-linked COFs with different molar ratios of 1,3,5-tris(4phormylphenyl)benzene and its fluorinated counterpart in the outer phenyl rings analogue 1,3,5tris(3-fluoro-4-phormylphenyl)benzene $\left(100 / 0,75 / 25,50 / 50,25 / 75\right.$, and 0/100). ${ }^{[53]}$ In this case, increasing the ratio of fluorinated to non-fluorinated monomers gave rise to an improvement in crystallinity and porosity. The BET surface areas of the mixed-linker COFs $(75 / 25,50 / 50,25 / 75)$ showed a linear increment from 710 to $1064 \mathrm{~m}^{2} \mathrm{~g}^{-1}$, while the $100 / 0$ monomer mixture displayed a BET surface area of $1802 \mathrm{~m}^{2} \mathrm{~g}^{-1}$. Quantum mechanical computational studies suggested this could be ascribed to the substantial energetic stabilization in the face-to-face stacking interaction of the electron poor fluorinated precursors in contrast to the electron rich non-fluorinated ones or even to the mixed linkers.

Salonen et al. have reported how the use of highly polarised aromatic monomers can have a positive effect on 2D COF crystallinity. ${ }^{[54]}$ 2,7-(Pyrene-4,5-dione)diboronic acid was allowed to react with 2,3,6,7,10,11-hexahydroxytriphenylene, giving rise to a highly crystalline boronate ester-linked COF (Dione-COF, Figure 8c) with a BET surface area of $1510 \mathrm{~m}^{2} \mathrm{~g}^{-1}$, which is almost twice the value for the analogue TP-COF with pyrene units instead of pyrene-4,5-dione units. The authors attributed the high order and large surface area to the antiparallel alignment of the pyrene-4,5-dione moiety that cancels out the net dipole moment. 
Another representative method to increase interlayer interactions and consequently COF crystallinity has been based on the incorporation of methoxy groups into the pore walls of iminelinked COFs (TPD-DMTP-COF, Figure $8 \mathrm{~d}) \cdot{ }^{[55]}$ In this case, the methoxy groups were introduced as substituents in the 2,5-positions of the terephthalaldehyde linker, which was condensed with 1,3,5tris(4-aminophenyl)benzene. These methoxy groups were able to delocalize the two lone pairs from the oxygen atoms over the aromatic phenyl linker, reinforcing the interlayer interaction and consequently increasing the crystallinity, BET surface area $\left(2105 \mathrm{~m}^{2} \mathrm{~g}^{-1}\right)$ and chemical stability of the material. Although this strategy has provided excellent results, it cannot be considered to be general. For instance, Banerjee and co-workers showed how the introduction of methoxy groups in a biphenyl linker decreased the crystallinity compared to the analogue imine COF without substituents, ${ }^{[56]}$ while another example from Jiang and co-workers showed that the introduction of methoxy groups led to amorphous samples. ${ }^{[18]}$

\subsection{Ionic interactions}

In some cases, the introduction of asymmetric ionic monomers has demonstrated to enhance COF order by inducing an alternating antiparallel monomer alignment to minimise charge repulsion. Jiang and colleagues combined a neutral node $(4,4$ ',4",4"'-(pyrene-1,3,6,8-tetrayl)tetraaniline) with a cationic linker (5,6-bis(4-formylbenzyl)-1,3-dimethyl-benzimidazolium) that produced a highly crystalline 2D COF (PyTTA-BFBIm-iCOF, Figure 9a). ${ }^{[42]}$ According to structural simulations, the high crystallinity, chemical stability and porosity (BET surface area of $1532 \mathrm{~m}^{2} \mathrm{~g}^{-1}$ ) were attributed to the cationic benzimidazolium moiety that was alternately oriented to each side of the pore walls. In addition, because of the presence of electrical dipoles on both sides, the uptake of $\mathrm{CO}_{2}$ was enhanced by three-fold compared to the neutral analogue. 
a)
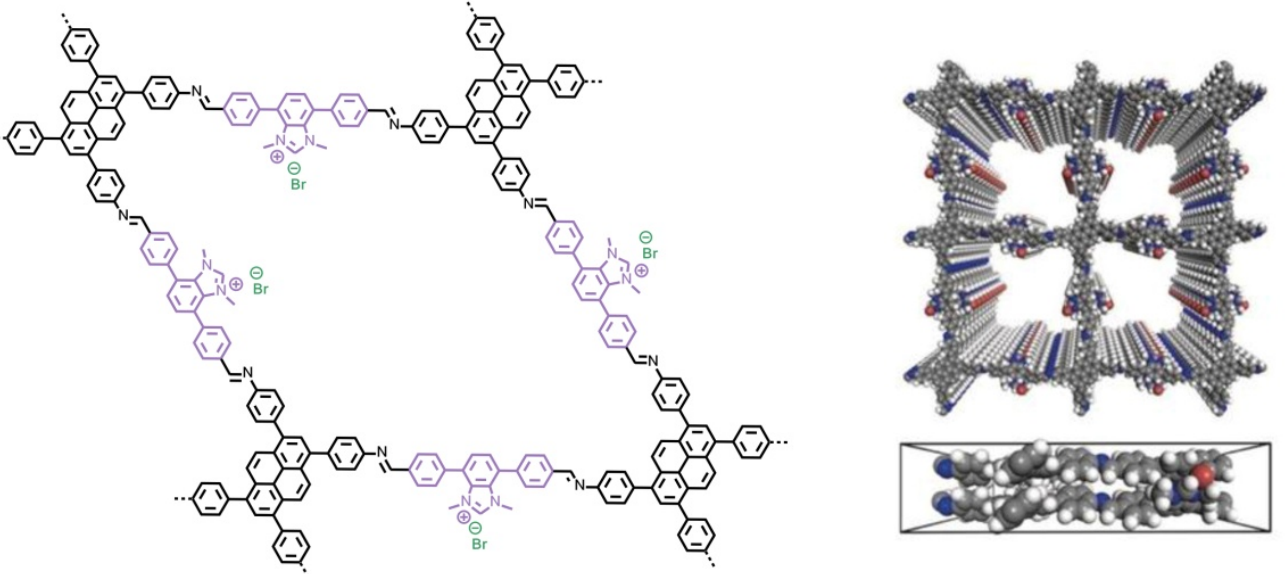

PyTTA-BFBIm-iCOF
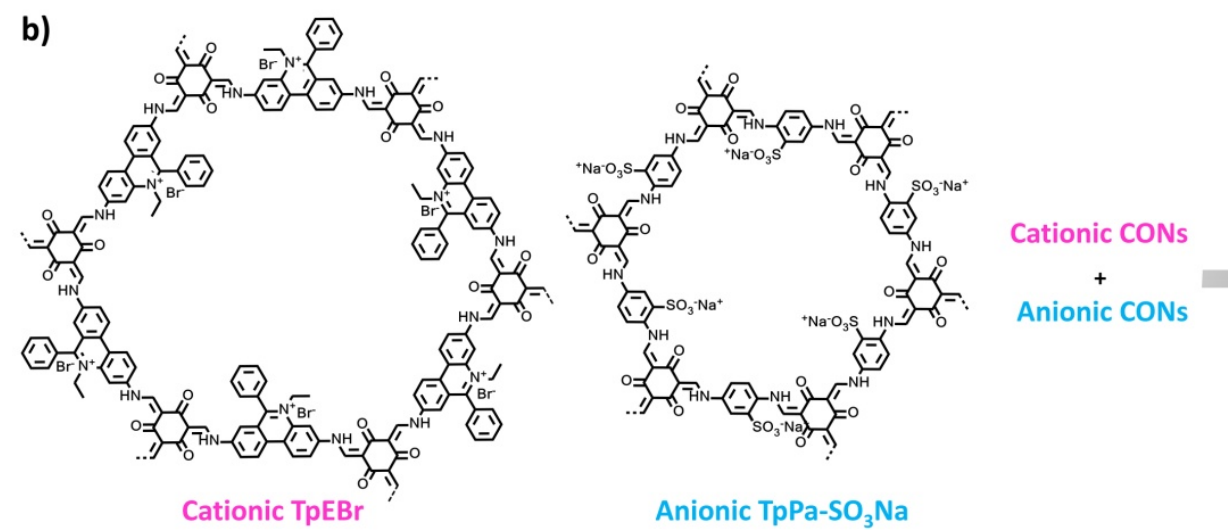

Figure 9. a) Chemical structure of PyTTA-BFBIm-iCOF with the top and side views of the reversed slipped AA-stacking mode. Adapted with permission. ${ }^{[42]}$ Copyright 2017, John Wiley and Sons. b) Chemical structure of the cationic $\mathrm{TpEBr}$ and anionic $\mathrm{TpPa}-\mathrm{SO}_{3} \mathrm{Na}$ and the representation of the compact dense structure with reduced apertures obtained by assembling the two kinds of iCONs under electrostatic interaction. Adapted with permission. ${ }^{[57]}$ Copyright 2020, American Chemical Society.

Alternatively, strong electrostatic interactions produced by combining opposite charges have been recently proved to increase COF crystallinity, and even to modulate the stacking and porosity. Zhao and co-workers mixed intrinsically charged cationic and anionic covalent organic nanosheets (Figure 9b), which assembled in a staggered packing with a dense structure as consequence of the strong electrostatic interactions. ${ }^{[57]}$ The layer-by-layer assembly gave rise to an ultrathin membrane with reduced aperture sizes that was suitable for $\mathrm{H}_{2} / \mathrm{CO}_{2}$ separation. 


\subsection{Hydrogen bonding interactions}

Intralayer hydrogen bonding has also demonstrated a positive effect in locking adjacent layers, which can increase the stability and crystallinity of COFs. Banerjee and co-workers have focused on a hydrogen bonded imine-linked COF (TpOMe-DAQ, Figure 10a) constructed from 2,4,6trimethoxy-1,3,5-benzenetricarbaldehyde and 2,6-diaminoanthraquinone, which exhibited ultrahigh chemical stability in drastic conditions: $\mathrm{H}_{2} \mathrm{SO}_{4}(18 \mathrm{M}), \mathrm{HCl}(12 \mathrm{M})$ and $\mathrm{NaOH}(9 \mathrm{M}) .{ }^{[58]}$ This strong stability was ascribed to the presence of $\mathrm{C}-\mathrm{H} \cdots \mathrm{N}$ hydrogen bonding between the methoxy $\mathrm{C}$ $\mathrm{H}$ and the imine of the adjacent layers, which protected the imine bond from being hydrolyzed. A similar approach led to COFs with high chemical stability for their potential application for sulfuric acid recovery. A series of imine-linked 2D COFs were obtained by condensing 2,4,6-trimethoxy1,3,5-benzenetricarbaldehyde with a set of six different amines, ${ }^{[59]}$ which allowed a comparison between 2D COFs that can undergo interlayer hydrogen bonding. The high stability was attributed again to the three methoxy groups in the nodes, which were almost perpendicularly to the layer, providing multiple $\mathrm{C}-\mathrm{H} \cdots \mathrm{N}$ hydrogen bonds between the imines of the adjacent layers.

Besides methoxy groups, interlayer $\mathrm{C}-\mathrm{H} \cdots \mathrm{N}$ hydrogen bonding within $2 \mathrm{D}$ COFs has been also observed with piperazine monomers. ${ }^{[60]}$ Yang and co-workers described an imine-linked COF (PDC-MA-COF, Figure 10b) obtained by reacting 1,4-piperazinedicarboxaldehyde and melamine, in which interlayer hydrogen bonding locked the adjacent layers and ensured a capacitive behaviour with an excellent cyclic stability.

Loh and co-workers have shown that, by modulating both intralayer and interlayer hydrogen bonding, it is possible to produce emissive hydrazone-linked 2D COFs. ${ }^{[61]}$ This series of 2D COFs, with a N-NH hydrogen bond donor and methoxy hydrogen bond acceptor in close proximity, allowed both intralayer and interlayer $\mathrm{C}-\mathrm{O} \cdots \mathrm{HN}$ hydrogen bonding that enhanced the solid-state 
photoluminescence of 2D COFs. In addition, the colour of the emission could be changed from blue to yellow and even white by introducing different side-chains. Loh and co-workers ${ }^{[62]}$ have also shown how the combination of interlayer and intralayer hydrogen bonding in 2D acylhydrazone COFs resulted in a rapid and gram-scale method for the synthesis of 2D COFs. Condensations of 1,3,5-triformylbenzene and 2,5-dialkyloxylterephthalohydrazide derivatives with different sidechain functionalities afforded highly homogeneous and crystalline 2D COFs (Tf-DHzOR COFs, Figure 10c) under open and stirring conditions in about 30 minutes, as confirmed by intense PXRD peaks. DFT calculations attributed this high crystallinity to the restriction of bond rotation by intramolecular hydrogen bonding, which enabled interlayer hydrogen bonding and dipole-dipole interactions, giving rise to antiparallel stacked configuration.

Recently, Smaldone and co-workers ${ }^{[63]}$ have developed a new class of $2 \mathrm{D}$ COFs, called COFamides, where amide hydrogen bonds reinforced interlayer interactions between layers. Two 2D COFs containing azine and imine (COFamide-1 and COFamide-2, Figure 10d) were synthesized by condensation of hydrazine or 1,4-phenylenediamine, respectively, with a triphenylarene aldehyde monomer containing secondary amides at the 1,3,5-positions of the central phenyl ring. As consequence of the non-planarity of the triphenylarene aldehyde platform, the amides were forced to be out of plane, giving rise to strong interlayer $\mathrm{N}-\mathrm{H} \cdots \mathrm{O}$ hydrogen bonding between the amide carbonyl oxygen of one layer and the amide hydrogen $(\mathrm{NH})$ in the adjacent layer. As a result, COFamide-1 and COFamide-2 showed sharp PXRD peaks and high BET surface areas (1271 and $1128 \mathrm{~m}^{2} \mathrm{~g}^{-1}$, respectively), which are more than the double than those obtained for the same monomers but without secondary amide groups. 


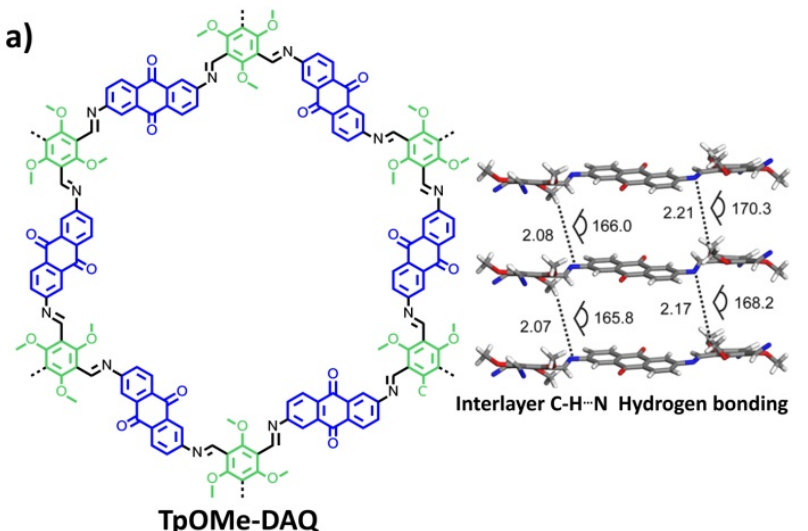

b)
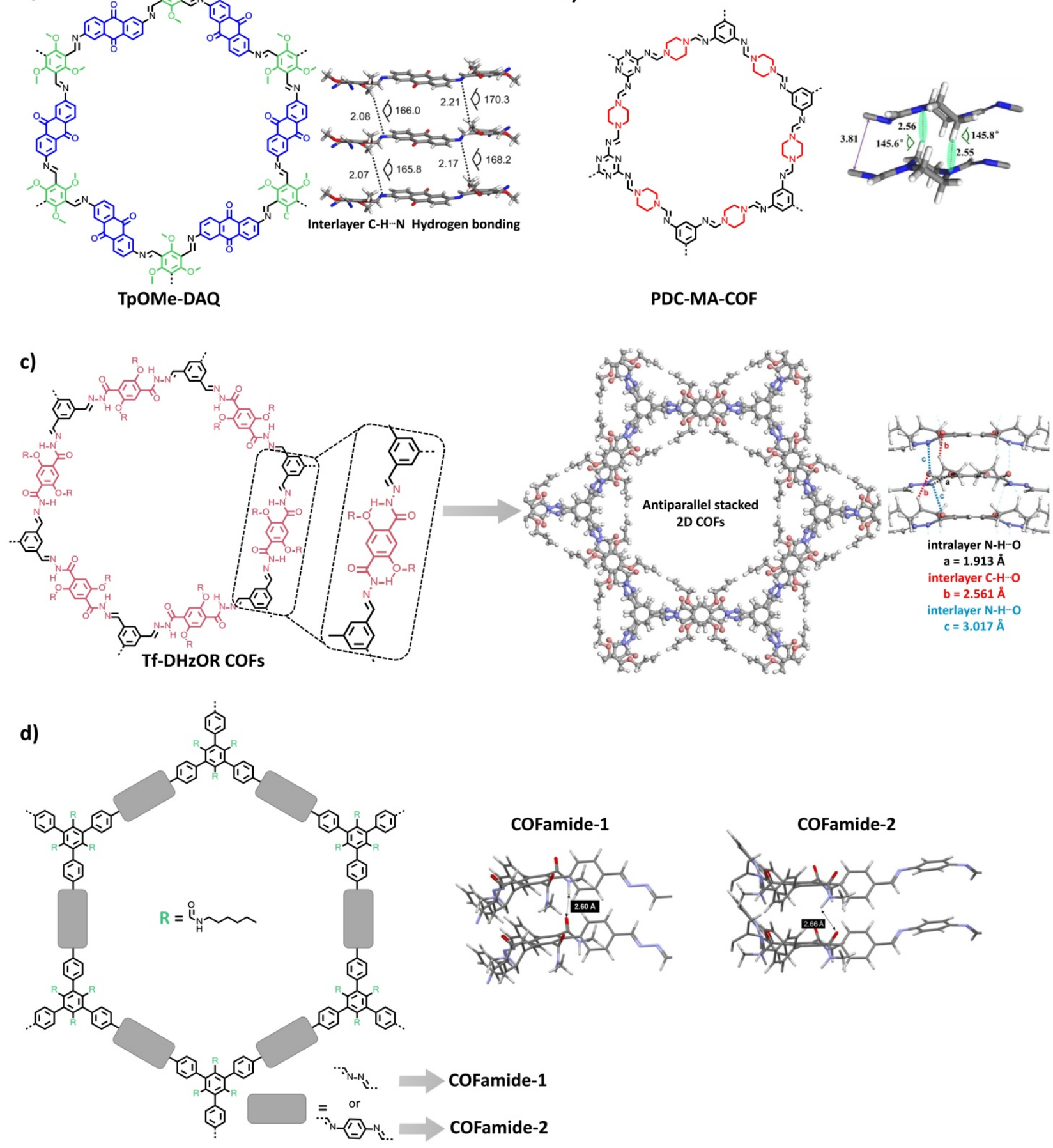

Figure 10. a) Structure of TpOMe-DAQ with the interlayer C-H $\cdots \mathrm{N}$ H-bonding in TpOMe-DAQ based on distances ( $\mathrm{H}$ to $\mathrm{N}$ atom; $\AA$ ) shown with dotted line and angles $(\mathrm{C}-\mathrm{H} \cdots \mathrm{N})$ in degree (layers spaced out for clear representation). Adapted with permission. ${ }^{[58]}$ Copyright 2018, American Chemical Society. b) Structure of PDC-MA-COF with its interlayer H-bonding structure. Adapted with permission. ${ }^{[60]}$ Copyright 2019, American Chemical Society. c) Structure of Tf-DHzOR COFs where the intramolecular hydrogen bonding restricts bond rotation, inducing an antiparallel stacking in which the intermolecular hydrogen bond further limits the random sliding of each of the COF layers. Adapted with permission. ${ }^{[62]}$ Copyright 2019 , American Chemical Society. d) Chemical structure of COFamide-1 and COFamide-2 with the view of the simulated structures of the eclipsed COFs along the a-axis. Adapted with permission. ${ }^{[63]}$ Copyright 2020, ChemRxiv. 


\section{Monomers Encoded with Disruptive Interactions}

The incorporation of monomers in 2D COFs with sterically-demanding substituents or with charges of the same sign can disrupt the interactions between layers, affecting crystallinity, layer arrangement, topology, interlayer distances, or even producing self-exfoliation.

\subsection{Steric interactions between monomers}

\subsubsection{Effects in crystallinity}

Generally, longer chain lengths produce a decrease in crystallinity. In an early study, Lavigne and co-workers reported a series of alkyl functionalised boronate ester-linked COFs (COF - $16 \AA$, COF

- $14 \AA, \mathrm{COF}-11 \AA$, Figure 11a) by condensation of benzene-1,3,5-triboronic acid with 2,6dialkylated-1,2,4,5-tetrahydroxybenzene (methyl, ethyl and propyl). Although the incorporation of those alkyl chains into the pore channels produced in all cases crystalline materials, the elongation of the chains gave rise to a decrease in crystallinity. ${ }^{[64]}$ On the contrary, the introduction of these hydrophobic alkyl groups increased the chemical stability, decreasing the hydrolysis rate of the boronate esters compared to the COF without substituents (COF - $18 \AA$, Figure 11a). ${ }^{[65]}$ Likewise, Uribe-Romo, Harper, Mendoza-Cortes and co-workers ${ }^{[66]}$ have described a family of $\beta$ ketoenamine-linked COFs functionalised with oligo-(ethylene oxide) chains with different lengths (TfpTP-R COFs, Figure 11b), showing the same gradual attenuation of crystallinity upon increasing the length of the side chains. 
a)

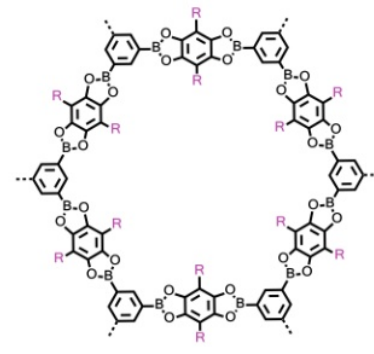

c)

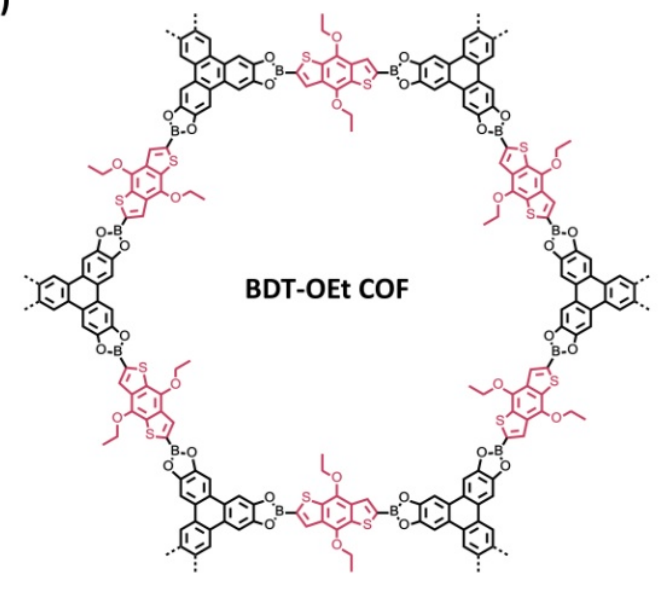

e)

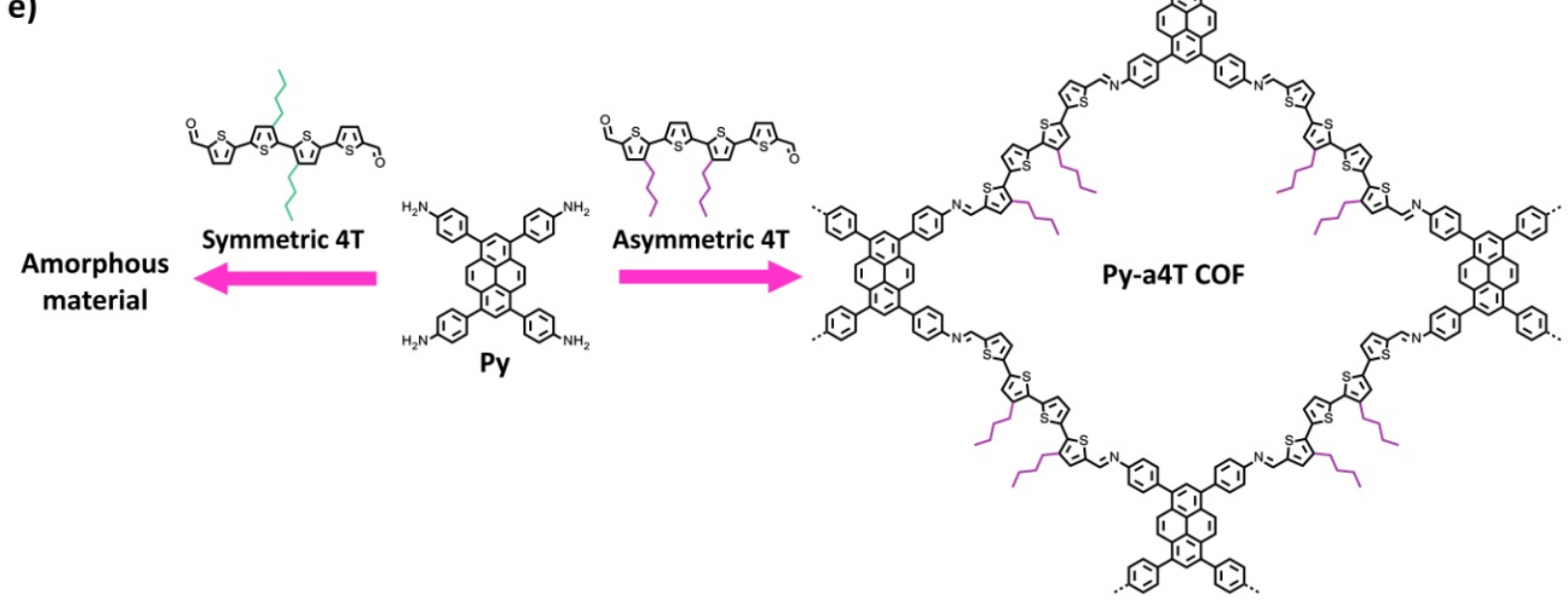

Figure 11. Structure of notable COFs with different sterically-demanding substituents: a) COF-18 $\AA$, COF-

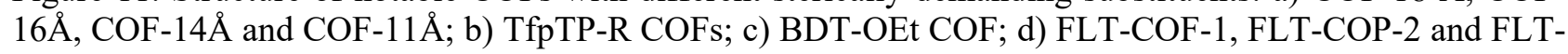
COP-3; and e) Py-a4T COF. 
Similarly, ethoxy side chains attached to benzodithiophene units gave rise to a highly crystalline COF by the condensation of 4,8-diethoxybenzo[1,2-b:4,5- $\left.b^{\prime}\right]$ dithiophene-2,6-diyldiboronic acid with hexahydroxytriphenylene (BDT-OEt COF, Figure 11c). ${ }^{[67]}$ In this case, the impact of the ethoxy chains was studied by developing a series of COFs containing mixtures of diethoxy substituted benzodithiophene units and the non-substituted one $(100 / 0,75 / 25,50 / 50,25 / 75$, and 0/100). The PXRD patterns broadened and smaller crystalline domains were observed upon the gradual incorporation of the diethoxy substituted monomer, while the layer arrangement and the interlayer distance were retained.

Smaldone and co-workers ${ }^{[68]}$ have observed how an increasing number of phenyl substituents in the 8,9-positions of the 7,10-diphenylfluoranthene affects the crystallinity of the resulting 2D COFs (Figure 11d). The planar fluoranthene monomer produced a crystalline 2D COF (FLT-COF-1) with strong diffraction and surface areas of $1180 \mathrm{~m}^{2} \mathrm{~g}^{-1}$, while the introduction of monomers with one or two phenyl rings in the fluoranthene core produced two-dimensional covalent organic polymers (2D COPs) (FLT-COP-2 and FLT-COP-3, respectively) with little diffraction and lower surface areas (555 and $515 \mathrm{~m}^{2} \mathrm{~g}^{-1}$, respectively). The differences in crystallinity and surface area were justified in terms of the additional out-of-plane phenyl substituents that hindered nucleation and crystallisation of the 2D COF structures.

Bein, Auras and co-workers ${ }^{[4]}$ have reported an example where the position of the alkyl chain in the same monomers can either disrupt completely the crystallinity leading to amorphous materials or provide crystalline 2D COFs. When dibutyl-quaterthiophene with two butyl chains located in symmetric positions (symmetric 4T) was allowed to react with Py, amorphous materials were obtained. However, when dibutyl-quaterthiophene with two butyl chains located in asymmetric positions (asymmetric 4T) was allowed to react with Py, a crystalline 2D COF was obtained (Pya4T COF, Figure 11e). Density functional theory simulations allowed concluding that symmetric 
linker would have the alkyl chains of adjacent layers very close, causing a considerable steric repulsion. On the other hand, the asymmetric unit would pack with the alkyl chains alternating on both sides, exhibiting a higher degree of long-range order.

\subsubsection{Effects in the arrangement between layers.}

Steric interactions can also influence the arrangement between layers in 2D COFs. In this regard, Yin and co-workers ${ }^{[69]}$ have reported two imine-linked COFs prepared with 2,4,6triformylphloroglucinol and 2,7-diaminofluorene or 9,9-dibutyl-2,7-diaminofluorene (COF-O-OH and $\mathrm{COF}-4-\mathrm{OH}$, respectively) that showed how the $n$-butyl groups changed the layer arrangement from an eclipsed $\mathrm{AA}$ to a staggered $\mathrm{AB}$ stacking mode (Figure 12a).

Cui, Liu and co-workers have demonstrated that introduction of either six ethyl or six isopropyl substituents in the non-planar $\mathrm{C}_{3}$-monomer 1,3,5-tris(4-aminophenyl)benzene produced 2D COFs with different packings. ${ }^{[70]}$ Condensation of triamine monomers (with and without ethyl or isopropyl substituents) with di- or trialdehydes resulted in a family $2 \mathrm{D}$ COFs with $\mathrm{AA}, \mathrm{AB}$ or $\mathrm{ABC}$ arrangements as an effect of the different interlayer steric hindrance (Figure 12b).

Zhao and co-workers ${ }^{[71]}$ have incorporated six methyl groups in a truxene-based building block (3,8,11-tri(4-formylphenyl)-5,5,10,10,15,15-hexamethyltruxene), generating a system where the methyl groups were out of the plane due to the tetrahedral configuration of the $\mathrm{sp}^{3}$ carbon. The condensation of this core with 1,4-diaminobenzene or benzidine led to the formation of iminelinked COFs (SIOC-COF-8 and SIOC-COF-9, respectively) with an inclined arrangement instead of the usual AA stacking (Figure 12c).

Zamora, Peréz, Platero-Prats and co-workers ${ }^{[72]}$ have described a series of imine-based 2D COFs (IMDEA-COF-1 and IMDEA-COF-2, Figure 12d) that showed how the steric repulsion between 
precursors in the nodes can lead to different packing modes. IMDEA-COF-1 obtained by condensation between 1,6-diaminopyrene (DAP) and 1,3,5-benzene-tricarbaldehyde (BTCA) showed an $\mathrm{AB}$ stacking. Meanwhile, IMDEA-COF-2 produced by condensation between 1,6diaminopyrene (DAP) and 2,4,6-triformylphloroglucinol (TP) exhibited an AA stacking. The authors justified these differences in terms of the steric repulsions between the pyrene and the hydroxyl group in TP that stabilized planar conformations, which led to the AA packing.

a)

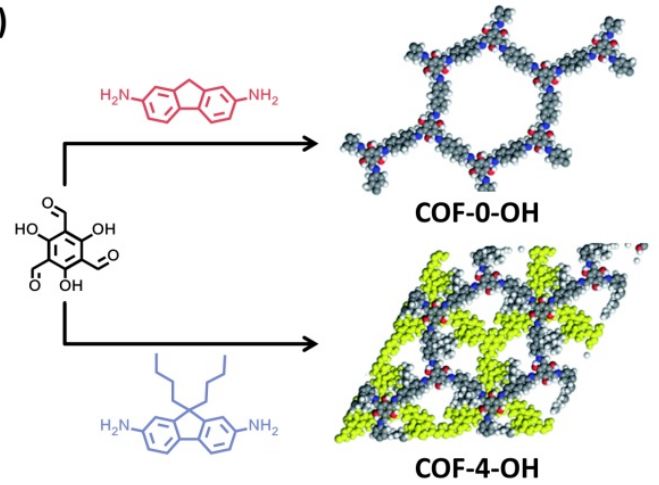

c)

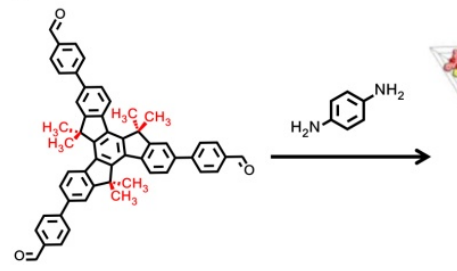

b)

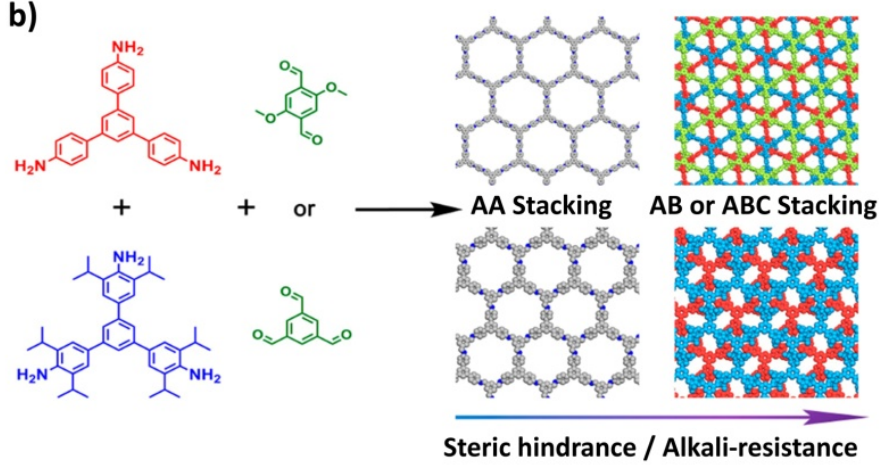

d)

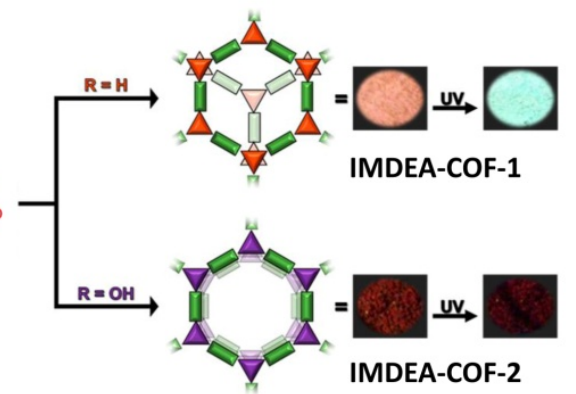

Figure 12. a) Representation of the formation of COF-0-OH and COF-4-OH with their packing modes. Adapted with permission. ${ }^{[69]}$ Copyright 2019, The Royal Society of Chemistry. b) Construction of the Binary, Ternary, and Quaternary COFs and their Stacking Modes. Reproduced with permission. ${ }^{[70]}$ Copyright 2018, American Chemical Society. c) Representation of the synthesis of SIOC-COF-8 with the packing mode. Adapted with permission. ${ }^{[71]}$ Copyright 2017, John Wiley and Sons. d) Schematic representation of the synthesis of IMDEA-COF-1 and IMDEA-COF-2, the packing modes and luminescence. Adapted with permission ${ }^{[72]}$ Copyright 2018, American Chemical Society.

\subsubsection{Effects on topology.}

Steric hindrance can also induce topology changes in COFs. Zhao and co-workers ${ }^{[73]}$ showed that when ETTA was condensed with 2,5-dihydroxyterephthalaldehyde (DHTA) a 2D COF with a kagome dual pore lattice (COF-DHTA, Figure 13a) was obtained. Oppositely, when reactions were carried out using 2,5-dialkoxy-terephathalaldehyde (ethoxy or butoxy) 2D COFs with a rhombus- 
like topology were obtained (COF-DETA and COF-DBTA, Figure 13a) as a consequence of the steric repulsion of the alkoxy chains in the triangular pore of the kagome lattice.

Wang, Sun and co-workers have reported the synthesis of COFs using biphenyl precursors with different substitution, showing how different degrees of rotation in the monomers can lead to COFs with different topologies. ${ }^{[74]}$ When 3,3',5,5'-tetra(p-aminophenyl)-biphenyl (BPTA), in which rotation along its longitudinal axis is permitted, was condensed with 1,2,4,5-tetrakis(4formylphenyl)-benzene (TPB-H), a 2D COF (2D-BPTA-COF, Figure 13b) was obtained. On the contrary, when the condensation was carried out with 3,3',5,5'-tetra( $p$-aminophenyl)-bimesitylene (BMTA), in which the methyl substituents locked a tetragonal conformation, a 3D COF (3DBMTA-COF, Figure 13b) was produced. 


\subsubsection{Effects on the interlayer distance}

Sterically-demanding substituents can diminish interlayer interactions. In this regard, Wang, Feng and co-workers prepared flexible films from alkoxy functionalized imine-linked COFs via interfacial polymerization by reacting 1,3,5-tris(4-aminophenyl)benzene with 2,5-dialkoxyterephthalaldehyde (methoxy, butoxy, hexyloxy and octyloxy), producing crystalline materials. ${ }^{[75]}$ Longer alkoxy chains led to longer interlayer distances as consequence of a less compact packing of the polymeric layers. Nevertheless, increasing the length of the alkoxy chains increased the resistance to humidity, which was ascribed to the hydrophobicity of the chains. The same effect was demonstrated in the aforementioned COF-O-OH and COF-4-OH, (see section 5.1.2, Figure 12a), showing an increase in the layer stacking distance of $1.76 \AA$ with $n$-butyl substituents (interlayer distance of 3.77 and $5.53 \AA$ for COF-O-OH and COF-4-OH, respectively). When $n$-octyl groups were introduced (COF-8-OH) even higher interlayer distances were observed $(6.42 \AA) .{ }^{69]}$

The introduction of triptycene nodes that sit perpendicular to the 2D COF plane disrupts completely interlayer $\pi-\pi$ stacking. El-Kaderi and co-workers ${ }^{[76]}$ have reported the synthesis of TD-COF-5 (Figure 13c), which was obtained by the co-polymerisation of hexahydroxytriptycene and 1,4benzenediboronic acid. TD-COF-5 exhibited a highly crystallinity and surface area $\left(3832 \mathrm{~m}^{2} \mathrm{~g}^{-1}\right)$ and a large interlayer distance of $7.4 \AA$. 
a)

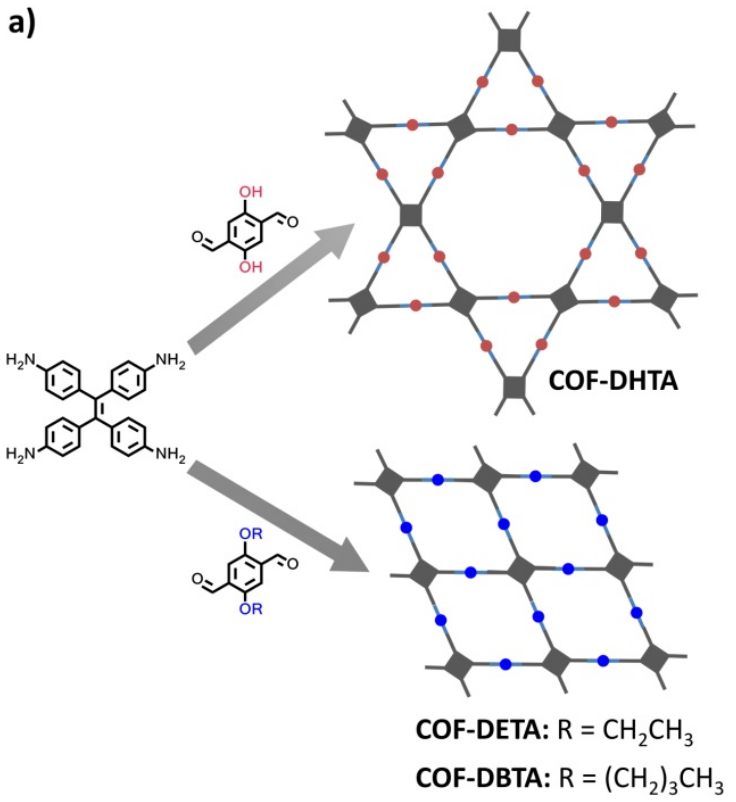

b)

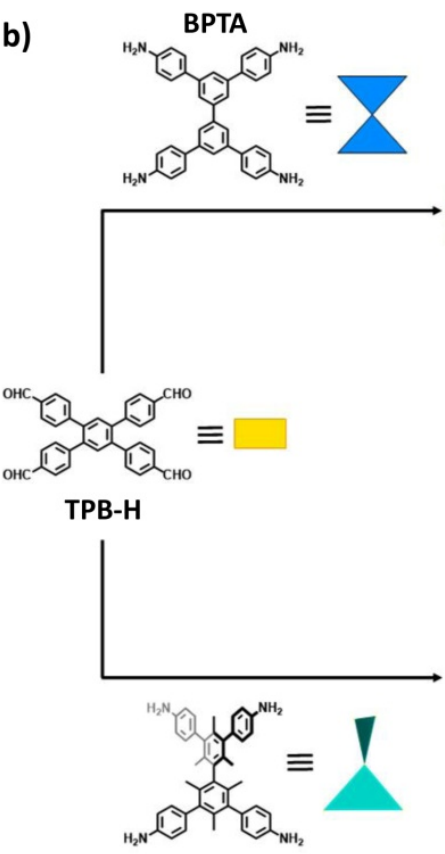

BMTA

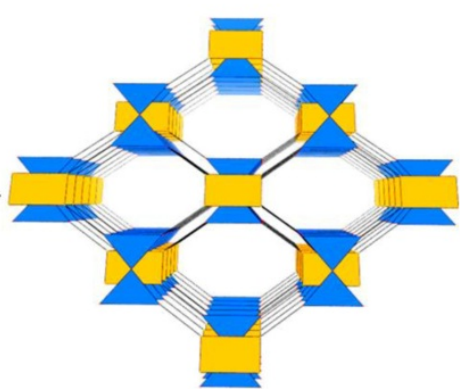

2D-BPTA-COF

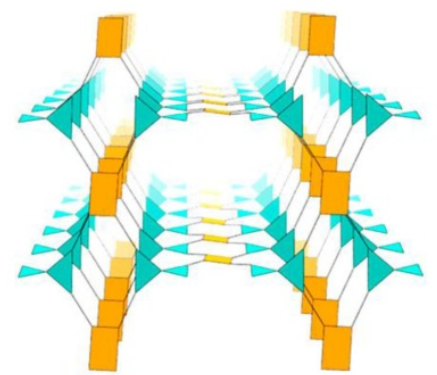

3D-BMTA-COF

c)

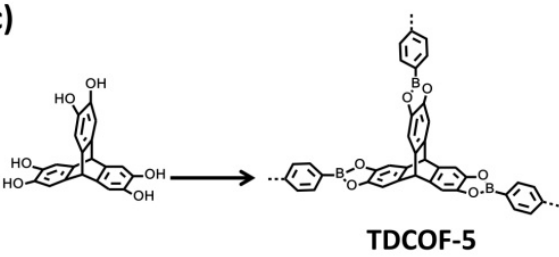

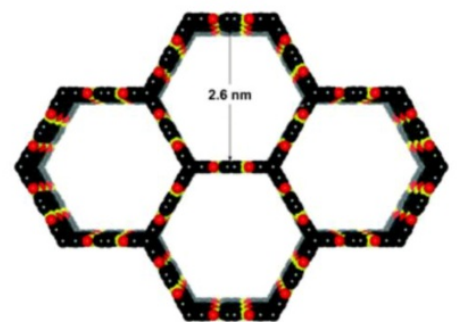

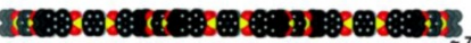
(6)

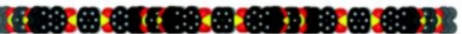

Figure 13. a) Synthesis of a single-pore COF (COF-DHTA) and dual-pore COFs (COF-DETA and COFDBTA) from ETTA and dialdehyde with different substituents. b) Schematic Representation of the Synthesis of 2D-BPTA-COF and 3D-BMTA-COF. Reproduced with permission. ${ }^{[74]}$ Copyright 2020, American Chemical Society. c) Synthesis of TDCOF-5 and space-filling models showing solid-state (AA) stacking; top-view and side-view. Adapted with permission. ${ }^{[76]}$ Copyright 2013, The Royal Society of Chemistry. 


\subsubsection{Self-exfoliation.}

In the previous sections, we have described how the effect of the bulky substituents can disturb the interlayer distances. If the steric interactions are sufficiently large, 2D COFs can spontaneously self-exfoliate into covalent organic nanosheets (CONs). Zhao and co-workers ${ }^{[77]}$ have reported triptycene nodes with two axial methyl groups in the 9,10-positions that were able to suppress interlayer interactions, yielding 2D COF monolayers in solution. Such self-exfoliating 2D COFs (2D Polymer-1 and 2D Polymer-2) were obtained by condensation of triptycene tricathecol with either 1,4-benzenediboronic acid or 4,4'-biphenyldiboronic acid, respectively. The resulting 2D COF monolayers were found to collapse in the formation of COF spheres.

Mateo-Alonso and co-workers have introduced a novel approach to exfoliate conjugated 2D polymers by introducing highly twisted hexaazatrinaphthylene nodes in the aromatic framework. ${ }^{[78]}$ This approach was based on the introduction of rigid acetylene moieties with triisopropylsilyl (TIPS) groups at the nodes of the framework, where the close proximity of the TIPS groups forced the aromatic framework to deviate from planarity, as there was not sufficient space to accommodate all the substituents in the same plane (TIPS-CMP, Figure 14a). Therefore, half of the TIPS groups were pushed above the plane of the framework and the other half below. The highly distorted aromatic framework with alternating out-of-plane substituents resulted in diminished interlayer interactions, thus favouring the exfoliation and dispersion of individual layers in organic media and leading to stable dispersions that could be processed into thin films.

A report from Beuerle and co-workers ${ }^{[79]}$ has described a $2 \mathrm{D}$ COF constituted of diketopyrrolopyrrole precursors with two 2-ethylhexyl groups and 5,10,15,20-tetrakis(4aminophenyl)porphyrin that produced microtubular structures (Figure 14b). The proposed mechanism involved the formation of small crystallites that evolved into larger sheet aggregates, 
which instead of crystallising because of the branched alkyl chains, spontaneously scrolled into tubular structures.

Cui and co-workers ${ }^{[80]}$ combined ETTA with the chiral binaphtyl linker BINOL (6,6'-dichloro-2,2'diethoxy-1,1'-binaphthyl-4,4'-dialdehyde) to produce CCOF7, a rhombus-shaped 2D COF with an slipped AA packing (Figure 14c). CCOF7 was easily exfoliated into 2D nanosheets through solvent-assisted liquid sonication, which was ascribed to the arrangement of the chiral BINOL with the twisted ETTA node that prevented effective layer packing. In fact, CCOF8 with the same BINOL monomer but a different trigonal node (1,3,5-tris(4-amino-3,5-diisopropylphenyl)benzene) led to a honeycomb lattice that could not be exfoliated, since the larger lattice and the ABC packing could accommodate the BINOL group in the structure without disturbing the interlayer packing. 

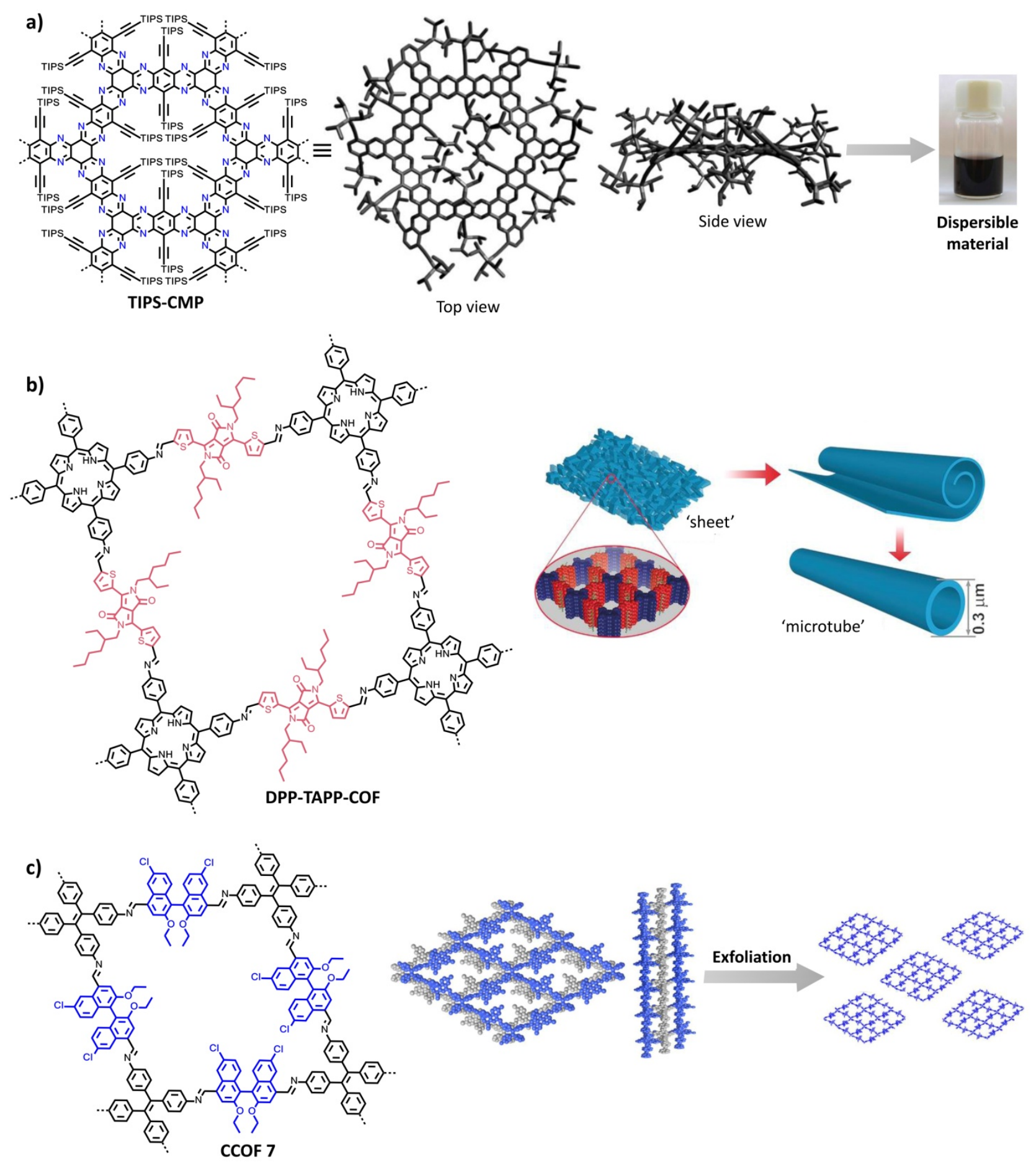

Figure 14. a) Chemical structure of TIPS-CMP, PM6-DH2 calculated structure of a closed pore of TIPS-CMP (top and side views) and dispersion of TIPS-CMP in TFA. Hydrogen atoms have been omitted from all structures for clarity. Adapted with permission. ${ }^{[78]}$ Copyright 2017, John Wiley and Sons; b) Chemical structure of DPP-TAPP-COF and the proposed self-assembly into microtubes. Adapted with permission. ${ }^{[79]}$ Copyright 2018, John Wiley and Sons. c) Structure of CCOF 7, view of the refined 2D crystal structure and schematic diagram for the exfoliation of CCOF 7 to ultrathin 2D nanosheets. Adapted with permission. ${ }^{[80]}$ Copyright 2019, American Chemical society. 


\subsection{Charged Monomers}

Generally, ionic monomers of the same sign make difficult $\pi-\pi$ stacking as consequence of charge repulsion, producing a decrease in crystallinity and chemical stability and allowing self-exfoliation and an easier processing. The introduction of charges in the framework can be also attained by protonation, ${ }^{[78,81]}$ however as this is not an inherent property of the monomers but rather of the media, and therefore it will not be discussed here.

Banerjee and co-workers ${ }^{[82]}$ have reported the introduction of positively charged guanidinium units, which provided TPGX COFs (Figure 15a) with different counter anions $\left(\mathrm{Cl}^{-}, \mathrm{Br}^{-}\right.$and $\left.\mathrm{I}^{-}\right)$. The presence of the loosely bound anions that disturbed $\pi$ - $\pi$ stacking and the positive charges on the framework resulted in self-exfoliation into 2D COF layered aggregates consisting of 3-6 layers. Pal, Sarkar and co-workers ${ }^{[83]}$ have also exploited this approach to produce self-exfoliating guanidinium-based 2D COFs by sonication.

Schlüter has reported the single crystal synthesis of positively charged $2 \mathrm{D}$ polymers by [2+2] cycloadditions (2D-P-1, Figure $15 \mathrm{~b}) .{ }^{[84]}$ The resulting 2D polymers were exfoliated by mechanical exfoliation, by stirring in $\gamma$-butyrolactone and by sonication with sodium cholate, maintaining in the first two cases an optimal degree of crystallinity, while in the case of sonication in the presence of the surfactant a loss of crystallinity was observed. ${ }^{[85]} 2 \mathrm{D}-\mathrm{P}-1$ could be transformed into a neutral pyridinic parent polymer (2D-P-2, Figure 15b) by exposure to gaseous ammonia, which could be exfoliated by sonication with sodium cholate with similar results in terms of quantity and quality to graphene.

Trabolsi and co-workers ${ }^{[86]}$ have described how the co-polymerisation of a viologen derivative with two different pyrene derivatives produced two viologen-based covalent organic polymers. When 
using a tetraaniline pyrene derivative (Py) few-layer covalent organic nanosheets were spontaneously formed. Meanwhile, in the case of a tetraaminoacetylene pyrene derivative the layers collapsed into tubular structures. Ma and co-workers ${ }^{[4]}$ have reported a viologen-based COF (PyVg-COF, Figure 15c) obtained by imine formation, which packed in an $\mathrm{AB}$ fashion. Remarkably, PyVg-COF could be exfoliated in solution into monolayers and multilayers in polar solvents with retention of crystallinity. These dispersions could be used to prepare high-quality, large area thin films. 
a)
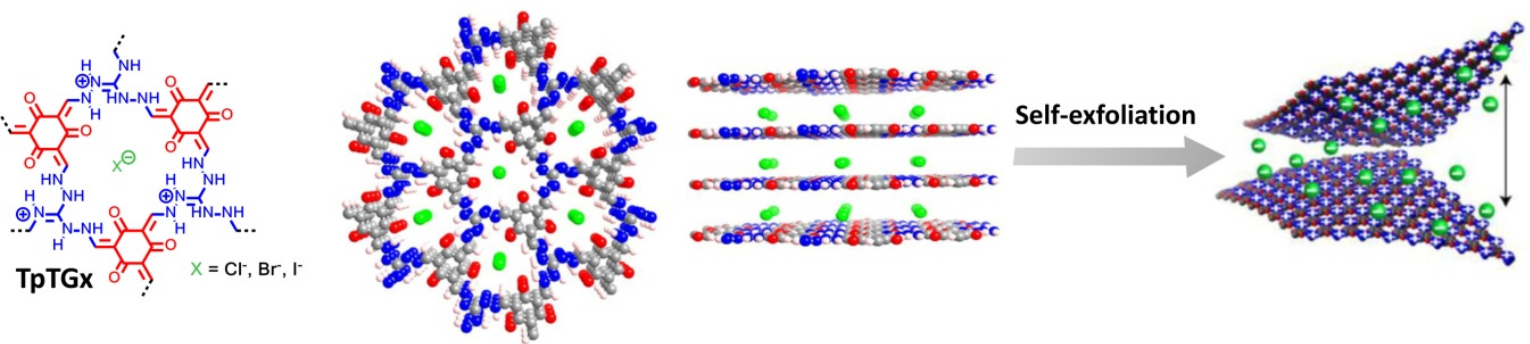

b)
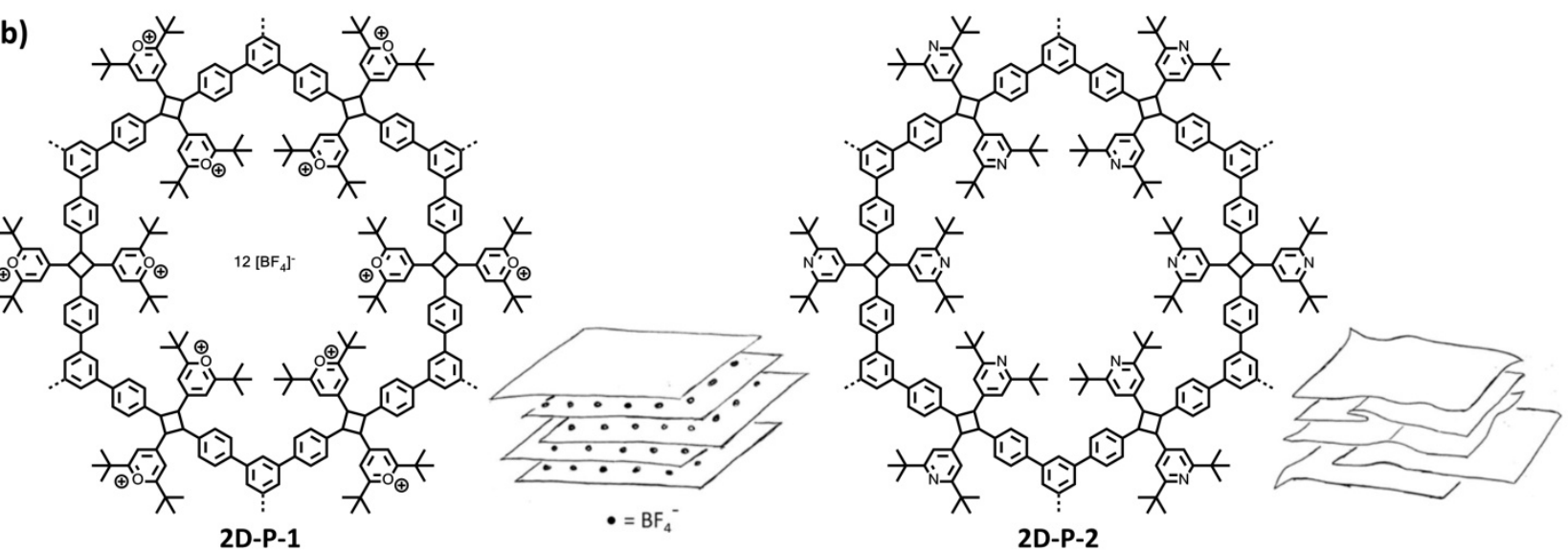

c)
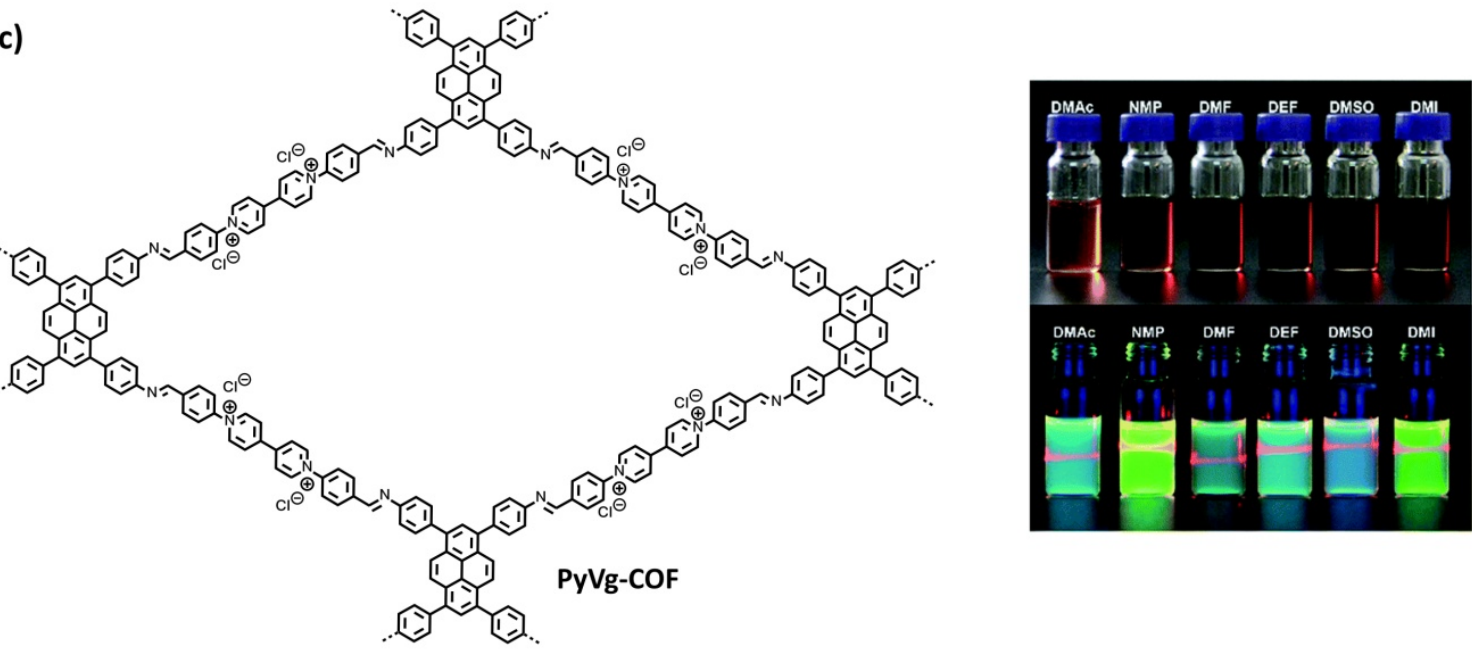

Figure 15. a) Structure of TpTGx, where $\mathrm{X}=\mathrm{Cl}-, \mathrm{Br}-, \mathrm{I}-$, ORTEP diagram and the modeling of TpTGCl in eclipsed mode and its stacking model showing individual layers. Adapted with permission. ${ }^{\left[{ }^{[2]}\right.}$ Copyright 2016, American Chemical society. b) Chemical structures of 2D-P-1 and 2D-P-2 with their layered structures. Adapted with permission. ${ }^{[85]}$ Copyright 2019, John Wiley and Sons. c) Chemical structures of PyVg-COF with the photos of the PyVg-COF dissolved in various solvents (top: concentrated solutions under sunlight; bottom: dilute solutions under $365 \mathrm{~nm}$ UV light showing the Tyndall effect). Adapted with permission. ${ }^{[4]}$ Copyright 2019, The Royal Society of Chemistry.

Another type of self-exfoliating covalent organic nanosheets are those reported by Ajayaghosh and co-workers, ${ }^{[87]}$ which were obtained from ethidium bromide precursors (EB-TFP-iCONs). These systems were exfoliated spontaneously in deionised water into bilayers due to the repulsive 
electrostatic interactions between layers. Ajayaghosh and co-workers ${ }^{[88]}$ have also described the synthesis of the parent propidium based 2D COFs with two positive charges per building block (PITFP-iCONs, Figure 16a), which led to a similar degree of self-exfoliation. Nevertheless thanks to the additional quaternary salts, PI-TFP-iCONs could be complexed with CB[7], which allowed the exfoliation and the re-stacking due to the reversible nature of such host-guest interaction.

Bai, Bai and co-workers ${ }^{[89]}$ have reported 2D COFs with piperazine substituents (Figure 16b) that are able to self-exfoliate into few layered ionic covalent organic nanosheets (iCONs) in polar organic solvents by coordination with $\mathrm{BF}_{3}$, which introduced ion pairs in the framework. Different morphologies, namely 2D sheets, multilayered aggregation or capsules were obtained in different polar solvents.

Xiang and co-workers ${ }^{[90]}$ have described a 2D COF constituted of fused iron quasi-phthalocyanine $\left(\mathrm{COF}_{\mathrm{BTC}}\right.$, Figure 16c), prepared by the polymerization of benzene-1,2,4,5-tetracarbonitrile in the presence of $\mathrm{FeCl}_{3}$. $\mathrm{COF}_{\mathrm{BTC}}$ was exfoliated in basic media as the $\mathrm{OH}^{-}$could penetrate between the layers, interacting with the Fe centers. As the dispersion becomes acidic, $\mathrm{COF}_{\mathrm{BTC}}$ would precipitate but it was able to redisperse again as soon as the $\mathrm{pH}$ was alkaline again. 
a)

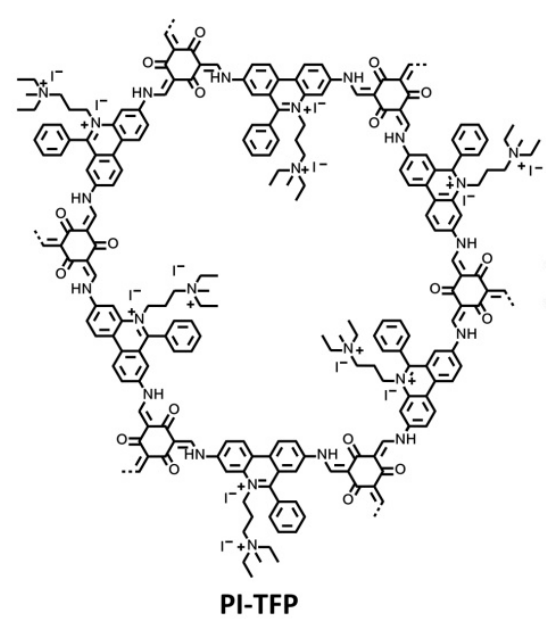

PI-TFP-iCONs

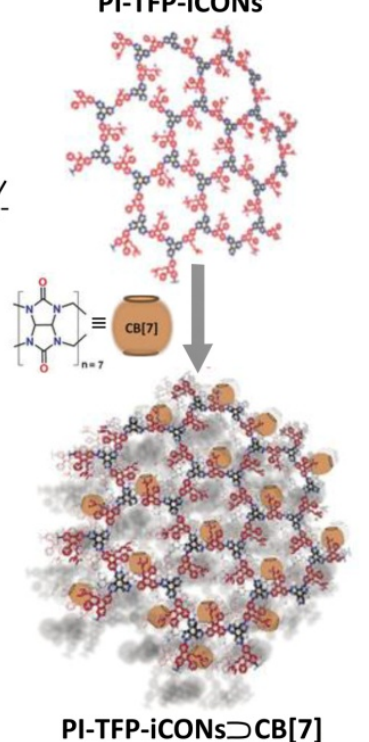

b)

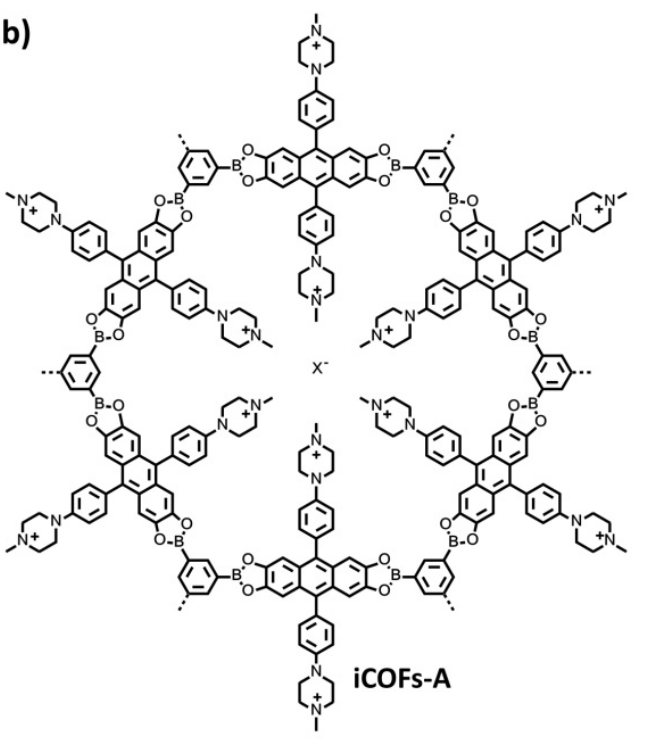

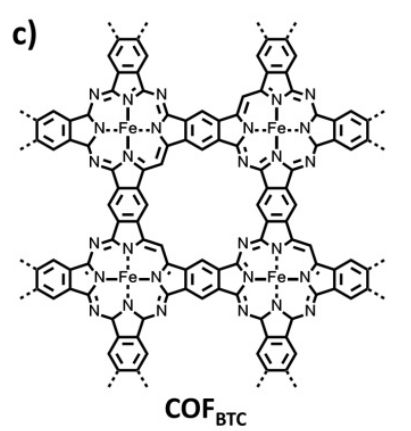

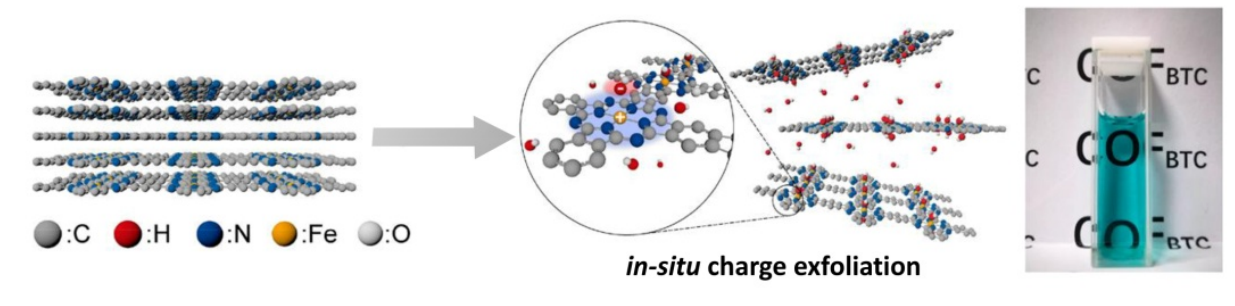

Figure 16. a) Chemical structure of PI-TFP with the schematic representation of CB[7]-mediated re-stacking of PI-TFP-iCONs. Adapted with permission. ${ }^{[88]}$ Copyright 2020, John Wiley and Sons. b) Chemical structure of iCOFs-A. c) Structure of $\mathrm{COF}_{\mathrm{BTC}}$ and representation of its exfoliation and dissolution route in alkaline media. A photo of the stable solution (at $\mathrm{pH}$ 13) is also provided. Adapted with permission ${ }^{[90]}$ Copyright 2019, American Chemical Society. 


\section{Conclusions}

In this Progress Report, we have brought up several examples that illustrate how the monomer structure can influence the interlayer interactions in 2D COF. For instance, the planarization of the monomers and layers - achieved by introducing planar monomers or intralayer hydrogen bonding motifs - has proven to be a very useful approach to increase interlayer $\pi-\pi$ stacking. Stackable monomers with persistent shapes have shown to be able to guide the stacking of COF layers during nucleation and growth, leading to an enhancement of crystallinity. The introduction of monomers with encoded attractive interactions - dipole, ionic and hydrogen bonding interactions - has also proven to increase the interlayer interactions, providing crystalline 2D COFs. Finally, the introduction of monomers with encoded disruptive interactions - steric and ionic interactions - can dramatically influence crystallinity, layer arrangement, topology, interlayer distances, and exfoliability of the resulting 2D COF.

Nevertheless, despite the extraordinary progress, the work in this area is not so extensive and more studies are necessary for developing general design rules that allow controlling the interactions between 2D COF layers. Our ability to design and synthesize virtually any monomer will continue to be a fundamental asset to influence and understand interlayer interactions. In addition, the exploration of other types linkages beyond the most studied boronate esters and imines will provide an additional knob to turn, since linkages can be directly involved in the interactions between 2D COF layers. The optimisation of reaction conditions and the development of general methods for the synthesis 2D COFs, although beyond the scope of this Progress Report, will also allow faster progress, since 2D COF synthesis is still a process of trial and error.

Overall, understanding interlayer interactions in 2D COFs will shine light on the chemistry beyond the single 2D COF layer. Also, controlling the arrangement between 2D COF layers will allow 
obtaining new properties from existing 2D COFs, preparing Van der Waals heterostructures from mixed 2D COFs or even hybrid Van der Waals heterostructures by combining 2D COFs with other 2D materials.

\section{Acknowledgements}

We acknowledge support of the Basque Science Foundation for Science (Ikerbasque), POLYMAT, the University of the Basque Country (Grupo de Investigación GIU17/054), Diputación Foral de Guipúzcoa (OF215/2016(ES)), Gobierno Vasco (PIBA 2019-09 and BERC program) and Gobierno de España (Ministerio de Economía y Competitividad CTQ2016-77970-R). This project has received funding from the European Research Council (ERC) under the European Union's Horizon 2020 research and innovation programme (grant agreement $\left.n^{\circ} 722951\right)$.

\section{References}

[1] A. P. Côté, A. I. Benin, N. W. Ockwig, M. O'Keeffe, A. J. Matzger, O. M. Yaghi, Science $2005,310,1166$.

[2] Introduction to Reticular Chemistry: Metal-Organic Frameworks and Covalent Organic Frameworks, Wiley-VCH, 2019; K. Sakaushi, M. Antonietti, Acc. Chem. Res. 2015, 48, 1591; N. Huang, P. Wang, D. Jiang, Nat. Rev. Mater. 2016, 1, 16068; J. L. Segura, M. J. Mancheño, F. Zamora, Chem. Soc. Rev. 2016, 45, 5635; J. Jiang, Y. Zhao, O. M. Yaghi, J. Am. Chem. Soc. 2016, 138, 3255; R. P. Bisbey, W. R. Dichtel, ACS Cent. Sci. 2017, 3, 533; Y. Jin, Y. Hu, W. Zhang, Nat. Rev. Chem. 2017, 1, 0056; J. Zhu, C. Yang, C. Lu, F. Zhang, Z. Yuan, X. Zhuang, Acc. Chem. Res. 2018, 51, 3191; S. B. Alahakoon, S. D. Diwakara, C. M. Thompson, R. A. Smaldone, Chem. Soc. Rev. 2020, 49, 1344.

[3] X. Feng, X. Ding, D. Jiang, Chem. Soc. Rev. 2012, 41, 6010; J. W. Colson, W. R. Dichtel, Nat. Chem. 2013, 5, 453; S.-Y. Ding, W. Wang, Chem. Soc. Rev. 2013, 42, 548; P. J. Waller, F. 
Gándara, O. M. Yaghi, Acc. Chem. Res. 2015, 48, 3053; C. S. Diercks, O. M. Yaghi, Science 2017, 355, eaal1585; S. Kandambeth, K. Dey, R. Banerjee, J. Am. Chem. Soc. 2019, 141, 1807; K. Geng, T. He, R. Liu, K. T. Tan, Z. Li, S. Tao, Y. Gong, Q. Jiang, D. Jiang, Chem. Rev. 2020, 10.1021/acs.chemrev.9b00550.

[4] S. M. J. Rogge, A. Bavykina, J. Hajek, H. Garcia, A. I. Olivos-Suarez, A. SepúlvedaEscribano, A. Vimont, G. Clet, P. Bazin, F. Kapteijn, M. Daturi, E. V. Ramos-Fernandez, F. X. Llabrés i Xamena, V. Van Speybroeck, J. Gascon, Chem. Soc. Rev. 2017, 46, 3134; F. Wang, X. Wu, X. Yuan, Z. Liu, Y. Zhang, L. Fu, Y. Zhu, Q. Zhou, Y. Wu, W. Huang, Chem. Soc. Rev. 2017, 46, 6816; H. Wang, Z. Zeng, P. Xu, L. Li, G. Zeng, R. Xiao, Z. Tang, D. Huang, L. Tang, C. Lai, D. Jiang, Y. Liu, H. Yi, L. Qin, S. Ye, X. Ren, W. Tang, Chem. Soc. Rev. 2019, 48, 488; S. Yuan, X. Li, J. Zhu, G. Zhang, P. Van Puyvelde, B. Van der Bruggen, Chem. Soc. Rev. 2019, 48, 2665; D. Rodríguez-San-Miguel, F. Zamora, Chem. Soc. Rev. 2019, 48, 4375; X. Liu, D. Huang, C. Lai, G. Zeng, L. Qin, H. Wang, H. Yi, B. Li, S. Liu, M. Zhang, R. Deng, Y. Fu, L. Li, W. Xue, S. Chen, Chem. Soc. Rev. 2019, 48, 5266; W. K. Haug, E. M. Moscarello, E. R. Wolfson, P. L. McGrier, Chem. Soc. Rev. 2020, 49, 839; Z. Wang, S. Zhang, Y. Chen, Z. Zhang, S. Ma, Chem. Soc. Rev. 2020, 49, 708 .

[5] J. L. Segura, S. Royuela, M. Mar Ramos, Chem. Soc. Rev. 2019, 48, 3903.

[6] S. J. Lyle, P. J. Waller, O. M. Yaghi, Trends Chem. 2019, 1, 172.

[7] S. Das, P. Heasman, T. Ben, S. Qiu, Chem. Rev. 2017, 117, 1515; J. Zhou, B. Wang, Chem. Soc. Rev. 2017, 46, 6927; J. Dong, X. Li, S. B. Peh, Y. D. Yuan, Y. Wang, D. Ji, S. Peng, G. Liu, S. Ying, D. Yuan, J. Jiang, S. Ramakrishna, D. Zhao, Chem. Mater. 2019, 31, 146; T. Ma, E. A. Kapustin, S. X. Yin, L. Liang, Z. Zhou, J. Niu, L.-H. Li, Y. Wang, J. Su, J. Li, X. Wang, W. D. Wang, W. Wang, J. Sun, O. M. Yaghi, Science 2018, 361, 48.

[8] J. Guo, Y. Xu, S. Jin, L. Chen, T. Kaji, Y. Honsho, M. A. Addicoat, J. Kim, A. Saeki, H. Ihee, S. Seki, S. Irle, M. Hiramoto, J. Gao, D. Jiang, Nat. Commun. 2013, 4, 2736; B. Zhang, M. Wei, H. Mao, X. Pei, S. A. Alshmimri, J. A. Reimer, O. M. Yaghi, J. Am. Chem. Soc. 2018, 140, 
12715; X. Guan, H. Li, Y. Ma, M. Xue, Q. Fang, Y. Yan, V. Valtchev, S. Qiu, Nat. Chem. 2019, $11,587$.

[9] B. J. Smith, N. Hwang, A. D. Chavez, J. L. Novotney, W. R. Dichtel, Chem. Commun. $2015,51,7532$.

[10] J. Dong, Y. Wang, G. Liu, Y. Cheng, D. Zhao, CrystEngComm 2017, 19, 4899.

[11] V. S. Vyas, F. Haase, L. Stegbauer, G. Savasci, F. Podjaski, C. Ochsenfeld, B. V. Lotsch, Nat. Commun. 2015, 6, 8508 .

[12] F. Haase, K. Gottschling, L. Stegbauer, L. S. Germann, R. Gutzler, V. Duppel, V. S. Vyas, K. Kern, R. E. Dinnebier, B. V. Lotsch, Mater. Chem. Front. 2017, 1, 1354.

[13] F. Haase, T. Banerjee, G. Savasci, C. Ochsenfeld, B. V. Lotsch, Faraday Discuss. 2017, 201, 247.

[14] A. F. M. El-Mahdy, C.-H. Kuo, A. Alshehri, C. Young, Y. Yamauchi, J. Kim, S.-W. Kuo, J. Mater. Chem. A 2018, 6, 19532.

[15] A. F. M. El-Mahdy, C. Young, J. Kim, J. You, Y. Yamauchi, S.-W. Kuo, ACS Appl. Mater. Interfaces 2019, 11, 9343.

[16] S. Kandambeth, D. B. Shinde, M. K. Panda, B. Lukose, T. Heine, R. Banerjee, Angew. Chem. Int. Ed. 2013, 52, 13052.

[17] D. B. Shinde, S. Kandambeth, P. Pachfule, R. R. Kumar, R. Banerjee, Chem. Commun. $2015,51,310$.

[18] X. Chen, M. Addicoat, E. Jin, L. Zhai, H. Xu, N. Huang, Z. Guo, L. Liu, S. Irle, D. Jiang, J. Am. Chem. Soc. 2015, 137, 3241.

[19] S. Kandambeth, V. Venkatesh, D. B. Shinde, S. Kumari, A. Halder, S. Verma, R. Banerjee, Nat. Commun. 2015, 6, 6786; A. Halder, S. Kandambeth, B. P. Biswal, G. Kaur, N. C. Roy, M. Addicoat, J. K. Salunke, S. Banerjee, K. Vanka, T. Heine, S. Verma, R. Banerjee, Angew. Chem. Int. Ed. 2016, 55, 7806.

[20] H.-L. Qian, C. Dai, C.-X. Yang, X.-P. Yan, ACS Appl. Mater. Interfaces 2017, 9, 24999. 
[21] X. Guo, Y. Tian, M. Zhang, Y. Li, R. Wen, X. Li, X. Li, Y. Xue, L. Ma, C. Xia, S. Li, Chem. Mater. 2018, 30, 2299.

[22] R. A. Maia, F. L. Oliveira, M. Nazarkovsky, P. M. Esteves, Cryst. Growth Des. 2018, 18, 5682.

[23] Z. Li, Y. Zhang, H. Xia, Y. Mu, X. Liu, Chem. Commun. 2016, 52, 6613.

[24] T. Li, X. Yan, Y. Liu, W.-D. Zhang, Q.-T. Fu, H. Zhu, Z. Li, Z.-G. Gu, Polym. Chem. 2020, $11,47$.

[25] J.-T. Yu, Z. Chen, J. Sun, Z.-T. Huang, Q.-Y. Zheng, J. Mater. Chem. 2012, 22, 5369.

[26] J.-R. Song, J. Sun, J. Liu, Z.-T. Huang, Q.-Y. Zheng, Chem. Commun. 2014, 50, 788.

[27] J. Zhen, S. Ding, J. Liu, Z. Huang, W. Wang, Q. Zheng, CrystEngComm 2016, 18, 1039.

[28] L. Ascherl, T. Sick, J. T. Margraf, S. H. Lapidus, M. Calik, C. Hettstedt, K. Karaghiosoff, M. Döblinger, T. Clark, K. W. Chapman, F. Auras, T. Bein, Nat. Chem. 2016, 8, 310.

[29] T. Sick, A. G. Hufnagel, J. Kampmann, I. Kondofersky, M. Calik, J. M. Rotter, A. Evans, M. Döblinger, S. Herbert, K. Peters, D. Böhm, P. Knochel, D. D. Medina, D. Fattakhova-Rohlfing, T. Bein, J. Am. Chem. Soc. 2018, 140, 2085.

[30] J. M. Rotter, S. Weinberger, J. Kampmann, T. Sick, M. Shalom, T. Bein, D. D. Medina, Chem. Mater. 2019, 31, 10008.

[31] N. Liu, L. Shi, X. Han, Q.-Y. Qi, Z.-Q. Wu, X. Zhao, Chin. Chem. Lett. 2019, 31, 386.

[32] A. C. Jakowetz, T. F. Hinrichsen, L. Ascherl, T. Sick, M. Calik, F. Auras, D. D. Medina, R. H. Friend, A. Rao, T. Bein, J. Am. Chem. Soc. 2019, 141, 11565.

[33] J. Wang, L. Si, Q. Wei, X. Hong, S. Cai, Y. Cai, ACS Appl. Nano Mater. 2018, 1, 132; F. Xu, S. Yang, X. Chen, Q. Liu, H. Li, H. Wang, B. Wei, D. Jiang, Chem. Sci. 2019, 10, 6001.

[34] P. Wang, Q. Xu, Z. Li, W. Jiang, Q. Jiang, D. Jiang, Adv. Mater. 2018, 30, 1801991.

[35] Y. Tian, S.-Q. Xu, C. Qian, Z.-F. Pang, G.-F. Jiang, X. Zhao, Chem. Commun. 2016, 52, 11704; Q. Gao, X. Li, G.-H. Ning, H.-S. Xu, C. Liu, B. Tian, W. Tang, K. P. Loh, Chem. Mater. $2018,30,1762$. 
[36] S. Dalapati, E. Jin, M. Addicoat, T. Heine, D. Jiang, J. Am. Chem. Soc. 2016, 138, 5797; M. Faheem, S. Aziz, X. Jing, T. Ma, J. Du, F. Sun, Y. Tian, G. Zhu, J. Mater. Chem. A 2019, 7, 27148. [37] F. Auras, L. Ascherl, A. H. Hakimioun, J. T. Margraf, F. C. Hanusch, S. Reuter, D. Bessinger, M. Döblinger, C. Hettstedt, K. Karaghiosoff, S. Herbert, P. Knochel, T. Clark, T. Bein, J. Am. Chem. Soc. 2016, 138, 16703.

[38] L. Ascherl, E. W. Evans, M. Hennemann, D. Di Nuzzo, A. G. Hufnagel, M. Beetz, R. H. Friend, T. Clark, T. Bein, F. Auras, Nat. Commun. 2018, 9, 3802.

[39] X. Chen, N. Huang, J. Gao, H. Xu, F. Xu, D. Jiang, Chem. Commun. 2014, 50, 6161.

[40] Y. Wu, H. Xu, X. Chen, J. Gao, D. Jiang, Chem. Commun. 2015, 51, 10096; B. Dong, L. Wang, S. Zhao, R. Ge, X. Song, Y. Wang, Y. Gao, Chem. Commun. 2016, 52, 7082; W. Leng, Y. Peng, J. Zhang, H. Lu, X. Feng, R. Ge, B. Dong, B. Wang, X. Hu, Y. Gao, Chem. Eur. J. 2016, 22, 9087; Z. Li, Y. Zhi, P. Shao, H. Xia, G. Li, X. Feng, X. Chen, Z. Shi, X. Liu, Appl. Catal. B 2019, 245, 334; H. Vardhan, L. Hou, E. Yee, A. Nafady, M. A. Al-Abdrabalnabi, A. M. Al-Enizi, Y. Pan, Z. Yang, S. Ma, ACS Sustain. Chem. Eng. 2019, 7, 4878.

[41] D. Bessinger, L. Ascherl, F. Auras, T. Bein, J. Am. Chem. Soc. 2017, 139, 12035.

[42] N. Huang, P. Wang, M. A. Addicoat, T. Heine, D. Jiang, Angew. Chem. Int. Ed. 2017, 56, 4982.

[43] S. Jin, T. Sakurai, T. Kowalczyk, S. Dalapati, F. Xu, H. Wei, X. Chen, J. Gao, S. Seki, S. Irle, D. Jiang, Chem. Eur. J. 2014, 20, 14608.

[44] N. Keller, D. Bessinger, S. Reuter, M. Calik, L. Ascherl, F. C. Hanusch, F. Auras, T. Bein, J. Am. Chem. Soc. 2017, 139, 8194.

[45] L. Wang, C. Zeng, H. Xu, P. Yin, D. Chen, J. Deng, M. Li, N. Zheng, C. Gu, Y. Ma, Chem. Sci. 2019, 10, 1023.

[46] Y. Meng, G. Lin, H. Ding, H. Liao, C. Wang, J. Mater. Chem. A 2018, 6, 17186. 
[47] M. Martínez-Abadía, C. T. Stoppiello, K. Strutynski, B. Lerma-Berlanga, C. Martí-Gastaldo,

A. Saeki, M. Melle-Franco, A. N. Khlobystov, A. Mateo-Alonso, J. Am. Chem. Soc. 2019, 141, 14403.

[48] Z. Xie, B. Wang, Z. Yang, X. Yang, X. Yu, G. Xing, Y. Zhang, L. Chen, Angew. Chem. Int. Ed. 2019, 58, 15742.

[49] N. Keller, T. Sick, N. N. Bach, A. Koszalkowski, J. M. Rotter, D. D. Medina, T. Bein, Nanoscale 2019, 11, 23338.

[50] X. Chen, M. Addicoat, S. Irle, A. Nagai, D. Jiang, J. Am. Chem. Soc. 2013, 135, 546.

[51] W. A. Braunecker, K. E. Hurst, K. G. Ray, Z. R. Owczarczyk, M. B. Martinez, N. Leick, A. Keuhlen, A. Sellinger, J. C. Johnson, Cryst. Growth Des. 2018, 18, 4160.

[52] S. B. Alahakoon, G. T. McCandless, A. A. K. Karunathilake, C. M. Thompson, R. A. Smaldone, Chem. Eur. J. 2017, 23, 4255.

[53] S. B. Alahakoon, G. Occhialini, G. T. McCandless, A. A. K. Karunathilake, S. O. Nielsen, R. A. Smaldone, CrystEngComm 2017, 19, 4882.

[54] L. M. Salonen, D. D. Medina, E. Carbó-Argibay, M. G. Goesten, L. Mafra, N. Guldris, J. M. Rotter, D. G. Stroppa, C. Rodríguez-Abreu, Chem. Commun. 2016, 52, 7986.

[55] H. Xu, J. Gao, D. Jiang, Nat. Chem. 2015, 7, 905.

[56] S. Chandra, S. Kandambeth, B. P. Biswal, B. Lukose, S. M. Kunjir, M. Chaudhary, R. Babarao, T. Heine, R. Banerjee, J. Am. Chem. Soc. 2013, 135, 17853.

[57] Y. Ying, M. Tong, S. Ning, S. K. Ravi, S. B. Peh, S. C. Tan, S. J. Pennycook, D. Zhao, J. Am. Chem. Soc. 2020, 142, 4472.

[58] A. Halder, M. Ghosh, A. Khayum M, S. Bera, M. Addicoat, H. S. Sasmal, S. Karak, S. Kurungot, R. Banerjee, J. Am. Chem. Soc. 2018, 140, 10941.

[59] A. Halder, S. Karak, M. Addicoat, S. Bera, A. Chakraborty, S. H. Kunjattu, P. Pachfule, T. Heine, R. Banerjee, Angew. Chem. Int. Ed. 2018, 57, 5797. 
[60] L. Li, F. Lu, R. Xue, B. Ma, Q. Li, N. Wu, H. Liu, W. Yao, H. Guo, W. Yang, ACS Appl. Mater. Interfaces 2019, 11, 26355.

[61] X. Li, Q. Gao, J. Wang, Y. Chen, Z.-H. Chen, H.-S. Xu, W. Tang, K. Leng, G.-H. Ning, J. Wu, Q.-H. Xu, S. Y. Quek, Y. Lu, K. P. Loh, Nat. Commun. 2018, 9, 2335.

[62] X. Li, J. Qiao, S. W. Chee, H.-S. Xu, X. Zhao, H. S. Choi, W. Yu, S. Y. Quek, U. Mirsaidov, K. P. Loh, J. Am. Chem. Soc. 2020, 142, 4932.

[63] S. B. Alahakoon, K. Tan, H. Pandey, G. T. McCandless, D. Grinffiel, A. Durand-Silva, T. Thonhauser, R. A. Smaldone, Chemxriv 2020, 10.26434/chemrxiv.12043368.v1.

[64] R. W. Tilford, S. J. Mugavero, P. J. Pellechia, J. J. Lavigne, Adv. Mater. 2008, 20, 2741.

[65] L. M. Lanni, R. W. Tilford, M. Bharathy, J. J. Lavigne, J. Am. Chem. Soc. 2011, 133, 13975.

[66] D. A. Vazquez-Molina, G. M. Pope, A. A. Ezazi, J. L. Mendoza-Cortes, J. K. Harper, F. J. Uribe-Romo, Chem. Commun. 2018, 54, 6947.

[67] M. S. Lohse, J. M. Rotter, J. T. Margraf, V. Werner, M. Becker, S. Herbert, P. Knochel, T. Clark, T. Bein, D. D. Medina, CrystEngComm 2016, 18, 4295.

[68] C. M. Thompson, G. Occhialini, G. T. McCandless, S. B. Alahakoon, V. Cameron, S. O. Nielsen, R. A. Smaldone, J. Am. Chem. Soc. 2017, 139, 10506.

[69] H.-Q. Yin, F. Yin, X.-B. Yin, Chem. Sci. 2019, 10, 11103.

[70] X. Wu, X. Han, Y. Liu, Y. Liu, Y. Cui, J. Am. Chem. Soc. 2018, 140, 16124.

[71] Y. Fan, Q. Wen, T.-G. Zhan, Q.-Y. Qi, J.-Q. Xu, X. Zhao, Chem. Eur. J. 2017, 23, 5668.

[72] P. Albacete, J. I. Martínez, X. Li, A. López-Moreno, S. a. Mena-Hernando, A. E. PlateroPrats, C. Montoro, K. P. Loh, E. M. Pérez, F. Zamora, J. Am. Chem. Soc. 2018, 140, 12922.

[73] Z.-F. Pang, T.-Y. Zhou, R.-R. Liang, Q.-Y. Qi, X. Zhao, Chem. Sci. 2017, 8, 3866.

[74] C. Gao, J. Li, S. Yin, J. Sun, C. Wang, J. Am. Chem. Soc. 2020, 142, 3718.

[75] P. Shao, J. Li, F. Chen, L. Ma, Q. Li, M. Zhang, J. Zhou, A. Yin, X. Feng, B. Wang, Angew. Chem. Int. Ed. 2018, 57, 16501. 
[76] Z. Kahveci, T. Islamoglu, G. A. Shar, R. Ding, H. M. El-Kaderi, CrystEngComm 2013, 15, 1524.

[77] T.-Y. Zhou, F. Lin, Z.-T. Li, X. Zhao, Macromolecules 2013, 46, 7745.

[78] A. B. Marco, D. Cortizo-Lacalle, I. Perez-Miqueo, G. Valenti, A. Boni, J. Plas, K. Strutyński, S. De Feyter, F. Paolucci, M. Montes, A. N. Khlobystov, M. Melle-Franco, A. MateoAlonso, Angew. Chem. Int. Ed. 2017, 56, 6946.

[79] B. Gole, V. Stepanenko, S. Rager, M. Grüne, D. D. Medina, T. Bein, F. Würthner, F. Beuerle, Angew. Chem. Int. Ed. 2018, 57, 846.

[80] X. Wu, X. Han, Q. Xu, Y. Liu, C. Yuan, S. Yang, Y. Liu, J. Jiang, Y. Cui, J. Am. Chem. Soc. 2019, 141, 7081.

[81] D. W. Burke, C. Sun, I. Castano, N. C. Flanders, A. M. Evans, E. Vitaku, D. C. McLeod, R. H. Lambeth, L. X. Chen, N. C. Gianneschi, W. R. Dichtel, Angew. Chem. Int. Ed. 2020, 59, 5165.

[82] S. Mitra, S. Kandambeth, B. P. Biswal, A. Khayum M, C. K. Choudhury, M. Mehta, G. Kaur, S. Banerjee, A. Prabhune, S. Verma, S. Roy, U. K. Kharul, R. Banerjee, J. Am. Chem. Soc. 2016, 138, 2823.

[83] H. Singh, M. Devi, N. Jena, M. M. Iqbal, Y. Nailwal, A. De Sarkar, S. K. Pal, ACS Appl. Mater. Interfaces 2020, 12, 13248.

[84] R. Z. Lange, G. Hofer, T. Weber, A. D. Schlüter, J. Am. Chem. Soc. 2017, 139, 2053.

[85] R. Z. Lange, K. Synnatschke, H. Qi, N. Huber, G. Hofer, B. Liang, C. Huck, A. Pucci, U. Kaiser, C. Backes, A. D. Schlüter, Angew. Chem. Int. Ed. 2020, 59, 5683.

[86] G. Das, T. Skorjanc, S. K. Sharma, T. Prakasam, C. Platas-Iglesias, D. S. Han, J. Raya, J.-C. Olsen, R. Jagannathan, A. Trabolsi, ChemNanoMat 2018, 4, 61.

[87] A. Mal, R. K. Mishra, V. K. Praveen, M. A. Khayum, R. Banerjee, A. Ajayaghosh, Angew. Chem. Int. Ed. 2018, 57, 8443.

[88] A. Mal, S. Vijayakumar, R. K. Mishra, J. Jacob, R. S. Pillai, B. S. Dileep Kumar, A. Ajayaghosh, Angew. Chem. Int. Ed. 2020, 10.1002/anie.201912363. 
[89] N. Zhang, T. Wang, X. Wu, C. Jiang, F. Chen, W. Bai, R. Bai, RSC Adv. 2018, 8, 3803.

[90] P. Peng, L. Shi, F. Huo, S. Zhang, C. Mi, Y. Cheng, Z. Xiang, ACS Nano 2019, 13, 878. 